\title{
1 Human embryoid bodies model basal lamina assembly and
}

2 muscular dystrophy

3

$4 \quad$ Alec R. Nickolls ${ }^{1,2}$, Michelle M. Lee ${ }^{1}$, Kristen Zukosky ${ }^{1,2}$, Barbara S. Mallon ${ }^{1}$,

5 Carsten G. Bönnemann ${ }^{1, *}$

6

$7 \quad{ }^{1}$ National Institute of Neurological Disorders and Stroke, National Institutes of Health,

8 Bethesda, MD 20892, USA

$9 \quad{ }^{2}$ Department of Neuroscience, Brown University, Providence, RI 02912, USA

$11{ }^{*}$ Correspondence: carsten.bonnemann@nih.gov 


\section{Abstract}

13

The basal lamina is a specialized sheet of dense extracellular matrix (ECM), linked to the plasma membrane of specific cell types in their tissue context, that serves as a structural scaffold for organ genesis and maintenance. Disruption of the basal lamina and its functions is central to many disease processes, including cancer metastasis, kidney disease, eye disease, muscular dystrophies, and specific types of brain malformation. The latter three pathologies occur in the dystroglycanopathies, which are caused by dysfunction of the ECM receptor dystroglycan. However, opportunities to study the basal lamina in various human disease tissues are restricted due to its limited accessibility. Here, we report the generation of embryoid bodies from human induced pluripotent stem cells to model basal lamina formation. Embryoid bodies cultured via this protocol mimic pre-gastrulation embryonic development, consisting of an epithelial core surrounded by a basal lamina and a peripheral layer of ECM-secreting endoderm. In dystroglycanopathy patient embryoid bodies, electron and fluorescence microscopy revealed ultrastructural basal lamina defects and reduced ECM assembly. By starting from patient-derived cells, these results establish a method for the in vitro synthesis of patient-specific basal lamina and recapitulate disease-relevant ECM defects seen in muscular dystrophies. Finally, we applied this system to evaluate an experimental ribitol supplement therapy on genetically diverse dystroglycanopathy patient samples. 


\section{Introduction}

34

Metazoan life relies on tissue compartmentalization to form ordered, discrete organs. This is partly accomplished by an extracellular matrix (ECM) barrier called the basal lamina, which ensheaths epithelial, endothelial, adipose, muscle, and nervous tissue [36]. The main components comprising the basal lamina are laminin isoforms, perlecan, nidogen, and collagen type IV, forming a complex lattice anchored to cell surface receptors [51]. This cell-ensheathing basal lamina is generally inter-connected, on its acellular matrix side, to a "lamina reticularis" composed of fibrillar collagens, microfibrils, and proteoglycans. Together, they form a multilayered basement membrane with tissue-specific mechanical properties [41, 51]. The terms "basal lamina" and "basement membrane" are sometimes used interchangeably. However, here they will refer to distinct structures, with the basal lamina being the dense, cell-attached component of the basement membrane.

The basal lamina is an essential structural component for organ genesis and maintenance. Its perturbation is linked to many human clinical conditions including metastatic cancer, nephropathy, lissencephaly, and muscular dystrophy. One mechanistic group of basal lamina-related diseases pertains to the dysfunction of the cell membrane ECM receptors integrin and dystroglycan [15, 47]. These receptors mediate cell attachment to the basal lamina, and in turn they influence the arrangement of the basal lamina itself $[20,30,31,35]$.

There is an expanding literature on the spectrum of disorders caused by dystroglycan receptor dysfunction, collectively termed the dystroglycanopathies, which 
56 are estimated to constitute roughly a third of all congenital muscular dystrophies [17]. A

57 hallmark of severe dystroglycanopathies is rupture or detachment of the basal lamina

58 that encases the brain and muscle fibers during development and structural

59 maintenance $[10,25]$. This specific combination of basal lamina abnormalities is

60

61

62

63

64

65

66

67

68 associated with a range of developmental nervous system malformations and progressive skeletal muscle degeneration that may ultimately be fatal [38].

The biochemical basis of the dystroglycanopathies is a reduction in a highly specific form of O-linked glycosylation on dystroglycan's a-subunit (aDG). This leads to a "hypoglycosylation" of the final aDG glycoepitope, which is referred to as the matriglycan [49]. Matriglycans on aDG confer binding activity to the ECM molecules laminin, perlecan, and nidogen [4]. Hypoglycosylated matriglycans have limited ECM binding capacity, which is thought to destabilize the basal lamina in muscle and brain tissue as a common disease pathway in the dystroglycanopathies [34, 35]. The 17 genes that are known to be mutated in the dystroglycanopathies all affect the formation of matriglycans and include various specific glycosyltransferases as well as enzymes preparing specific sugars to be incorporated, while only very few cases involve the gene encoding dystroglycan itself. Based on this knowledge, a large proportion of the dystroglycanopathy cases can now be clarified genetically [8, 17].

Understanding the mechanisms of pathogenesis and developing rational therapies for the dystroglycanopathies remains a challenge, in part due to its phenotypic and genetic heterogeneity. A large collection of dystroglycanopathy animal models recapitulates many aspects of the clinical spectrum [38]. However, such approaches fall 
78 short of modeling the genetic diversity of human patients for assessing disease

79 phenotypes and drug responses.

To study patient-specific basal lamina in a model system, we developed a

81 protocol to generate ECM-containing spheroids from human induced pluripotent stem

82 cells (hiPSCs), which we refer to as embryoid bodies. hiPSC-derived embryoid bodies

83 produce their own basal lamina and represent a simplified 3D system to investigate

84 human ECM and its receptors in diverse genetic contexts. As a proof of concept, we

85 applied this method to produce embryoid bodies from a variety of dystroglycanopathy

86 patients. We observed subtle basal lamina defects that correlated with disease severity

87 and corroborate findings in mouse models. Lastly, we evaluated patient hiPSCs and

88 embryoid bodies treated with the sugar alcohol ribitol, a recently proposed therapeutic

89 for the dystroglycanopathies. By correlating patient genotype and drug response, this

90 approach allows for pre-clinical prediction of therapeutic efficacy in specific individuals. 


\section{Materials and methods}

\section{Generation of hiPSC Lines}

94 Written informed consent for patient participation was obtained by a qualified 95 investigator (protocol 12-N-0095 approved by the National Institute of Neurological 96 Disorders and Stroke, National Institutes of Health). Dystroglycanopathy patient hiPSCs

97 were reprogrammed from dermal fibroblasts using an hOKSML mRNA reprogramming 98 kit (Stemgent, 00-0067). Control-1 hiPSCs were reprogrammed in the same manner 99 from BJ foreskin fibroblasts (ATCC, CRL-2522). Immunocytochemical validation of germ 100 layer differentiation was performed off-site (Stemgent). Control-2 hiPSCs were 101 reprogrammed from control foreskin fibroblasts (ATCC, CRL-2097) using the CytoTune102 iPS 2.0 Sendai reprogramming kit (Thermo Fisher, A16517). Control-3 hiPSCs (NC15) 103 were previously generated by lentiviral reprogramming of adult dermal fibroblasts [18]. 104 Karyotype analysis was performed after at least 10 passages (WiCell), and all cell lines 105 were routinely tested for mycoplasma contamination (LT07-118, Lonza).

hiPSC Culture

108 Human hiPSCs were maintained with daily changes of E8 medium (Thermo Fisher, 109 A1517001) on tissue culture-treated polystyrene plates coated with Matrigel (Corning, 110 354277) and passaged every 4 - 6 days using ReLeSR (STEMCELL Technologies, 111 05872). 
114 Differentiation of human hiPSC-derived embryoid bodies was performed essentially as 115 described previously for human embryonic stem cells [45]. At least one passage before 116 differentiation, hiPSCs were transitioned to MEF co-culture. The MEFs (Millipore, 117 PMEF-CF) were seeded at 30,000 cells $/ \mathrm{cm}^{2}$ on plates coated with gelatin (STEMCELL 118 Technologies, 07903) and maintained in serum-containing medium consisting of KO119 DMEM (10829-018), 20\% FBS (26140-079), $100 \mu \mathrm{M}$ non-essential amino acids (11140120 050), 2 mM GlutaMAX (35050-061), and $55 \mu \mathrm{M} \beta$-mercaptoethanol (21985-023) (all 121 from Invitrogen). hiPSCs were dissociated with ReLeSR and plated on MEFs in knockout serum 123 replacement medium consisting of KO-DMEM, 20\% KSR (Invitrogen, 10828-028), 100 $124 \mu \mathrm{M}$ non-essential amino acids, $2 \mathrm{mM}$ GlutaMAX, $55 \mu \mathrm{M} \beta$-mercaptoethanol, $10 \mathrm{ng} / \mathrm{mL}$ 125 bFGF (Thermo Fisher, 233-FB-025), and $10 \mu \mathrm{M}$ Y-27632 (Tocris, 1254). The medium 126 was changed daily (without Y-27632) until hiPSCs reached roughly 60\% confluency. For embryoid body formation, hiPSCs were dissociated by Collagenase Type IV 128 (Invitrogen 17104-019) and manual scraping followed by gravity sedimentation to 129 remove as many MEFs as possible. The cells were then individualized with Accutase 130 (Invitrogen, A1110501), and $2.4 \times 10^{6}$ cells were seeded per well of an AggreWell 400 131 (STEMCELL Technologies, 34411) following manufacturer's instructions by 132 centrifugation in X-VIVO 10 medium (Lonza, 04-380Q) with $10 \mu \mathrm{M} \mathrm{Y}-27632$. The 133 following day, spheroids were extracted from the AggreWell 400 according to 134 manufacturer's instructions and cultured in ultra-low attachment dishes (Corning, 3262) 135 with serum-containing medium for up to four days with a medium change every other 136 day. 
138

139

140

141

142

143

144 145

146

147

148

149

150

151

152

153

154

155

156

157

158 159

\section{Endoderm-Free Embryoid Body Culture}

Before endoderm-free embryoid body experiments, feeder-free hiPSCs were maintained on Matrigel in E8 medium. hiPSCs were dissociated with Accutase, and 1.2 $\times 10^{6}$ cells were seeded per well of an AggreWell 400 by centrifugation in E8 with $10 \mu \mathrm{M}$ Y-27632. The next day, spheroids were transferred to ultra-low attachment 6-well plates (Corning, 3471) in E8 supplemented with laminin (Invitrogen, 23017015) for 48 hours. $140 \mu \mathrm{g} / \mathrm{mL}$ laminin was used except where otherwise stated in the main text.

\section{Western Blotting}

hiPSCs in $100 \mathrm{~mm}$ dishes were lysed by $200 \mu \mathrm{L}$ RIPA buffer with protease and phosphatase inhibitors. $1 \mathrm{mg}$ soluble protein was incubated overnight at $4{ }^{\circ} \mathrm{C}$ in $500 \mu \mathrm{L}$ RIPA with $50 \mu \mathrm{L}$ agarose-bound wheat germ agglutinin (Vector Labs, AL-1023) to enrich the glycoprotein fraction. The agarose was washed three times with RIPA, and the glycoproteins were eluted by 5 -minute incubation at $95^{\circ} \mathrm{C}$ in SDS-PAGE loading buffer. Glycoproteins were ran on $4-12 \%$ Bis-Tris gels and transferred to PVDF membranes.

All blocking steps and antibody incubations were performed in TBST with $5 \%$ milk (glyco-aDG and $\beta D G$ ) or $5 \%$ donkey serum (core-aDG). The membranes were probed with antibodies against glyco-aDG, core-aDG, or $\beta D G$ overnight at $4{ }^{\circ} \mathrm{C}$. Labeling was visualized by chemiluminescence with appropriate secondary HRPconjugated antibodies on a ChemiDoc XRS+ (Bio-Rad). See Table 2 for a list of antibodies used in this study. 

microscope.

\section{Laminin Overlay Assay}

PVDF membranes were first blocked with 5\% milk in laminin binding buffer (LBB; 140 $\mathrm{mM} \mathrm{NaCl}, 10 \mathrm{mM}$ triethanolamine, $1 \mathrm{mM} \mathrm{CaCl}_{2}, 1 \mathrm{mM} \mathrm{MgCl}_{2}, 0.05 \%$ Tween, $\mathrm{pH}$ 7.6) and then incubated with $1 \mu \mathrm{g} / \mathrm{mL}$ laminin in LBB overnight at $4{ }^{\circ} \mathrm{C}$. PVDF membranes were washed and probed with laminin antibodies for 1 hour at room temperature in LBB with $5 \%$ milk. The membranes were then washed and probed with an appropriate HRPconjugated secondary antibody for 1 hour at room temperature in LBB with $5 \%$ milk before chemiluminescent imaging.

\section{Immunofluorescence Microscopy}

For immunocytochemistry, cells in chamber slides were fixed for 10 minutes in $4 \%$ PFA and then washed with PBS before staining. For immunohistochemistry, embryoid bodies were fixed for 20 minutes in 4\% PFA, cryoprotected by overnight incubation with $30 \%$ sucrose in PBS, and frozen in optimum cutting temperature (OCT) compound (VWR, 25608-930). OCT blocks were sectioned at $10 \mu \mathrm{m}$ thickness on a cryostat and mounted on slides for staining.

Slides were blocked in $10 \%$ goat serum and $0.1 \%$ Triton $\mathrm{X}-100$ for 1 hour at room temperature before primary antibody incubation with 3\% goat serum overnight at 4 ${ }^{\circ} \mathrm{C}$. Secondary antibody labeling was performed at room temperature for 1 hour. Refer to Table 2 for antibody dilutions and catalog numbers. Fluorescent images were captured on a Leica TSC SP5 II confocal microscope or a Nikon Eclipse Ti-E inverted 


\section{Transmission Electron Microscopy}

184 Embryoid bodies were fixed for 30 minutes at room temperature in $0.1 \mathrm{M}$ cacodylate 185 buffer with $4 \%$ glutaraldehyde, $\mathrm{pH}$ 7.4. Samples were then coated in agarose, washed 186 with buffer, and incubated for 60 minutes at $4{ }^{\circ} \mathrm{C}$ in $0.1 \mathrm{M}$ cacodylate buffer with $1 \%$ 187 osmium tetroxide, $\mathrm{pH}$ 7.4. The samples were washed and stained en bloc overnight at 4 $188{ }^{\circ} \mathrm{C}$ in $0.1 \mathrm{M}$ acetate buffer with $1 \%$ uranyl acetate, $\mathrm{pH} 5.0$. The next day, samples were 189 dehydrated in ethanol and epoxy resin embedded. $70 \mathrm{~nm}$ sections were cut and 190 counterstained with lead citrate and uranyl acetate. Micrographs were captured on a 191 JEOL1200EX transmission electron microscope with a digital CCD camera (AMT XR192 100, Danvers, MA, USA).

\section{Image Quantification and Statistics}

195 The image processing program Fiji was used to analyze all western blots and 196 microscopy images. Staining intensity was measured by drawing equal sized regions of 197 interest and measuring the average pixel intensity in each sample. Before statistical 198 measurements, all data were assessed for normality using the Shapiro-Wilk test. If data 199 were not normally distributed, they were analyzed by Kruskal-Wallis test with Dunn's 200 correction for multiple comparisons (Fig. 5e). If data were normally distributed, an 201 ordinary one-way ANOVA was used and corrected with Tukey's multiple comparisons 202 test (all other figures). The number of replicates, $\mathrm{n}$, for each analysis are reported in 203 their respective figure legend. Prism 7.0 (GraphPad software) was used to make all 204 statistical tests and graphs. 
205

206

207

208

209

210

211

212

213

214

215

216

217

218

219

220

221

222

223

224

225

226

227

\section{Results}

\section{Human Embryoid Bodies Mimic Pre-Gastrulation Development}

To establish an hiPSC-based model of basal lamina assembly, we used a microwell plate to generate spheroids of human hiPSCs (Fig. 1a, b). Following transfer of the spheroids into suspension culture, we tested multiple conditions for optimal ECM production. Spheroids grown in a standard knockout serum replacement (KSR) medium formed a cavitated core and differentiated into a Nestin+ neuroectodermal lineage (Fig. 2a). This result could be achieved with either feeder-free hiPSCs or with feederdependent hiPSCs, the latter of which were cultured on a feeder layer of mouse embryonic fibroblasts (MEFs) prior to spheroid formation.

We next tested the effect of serum on hiPSC spheroid differentiation. In a medium with $20 \%$ serum, spheroids from feeder-free hiPSCs consisted of an outer SOX17+ endodermal layer and a disorganized core of differentiated OCT4- cells (Fig. 2b). These spheroids, as well as those discussed above that were maintained in KSR medium, were mostly devoid of ECM as assessed by antibodies against the basal lamina protein laminin (data not shown).

In contrast, we found that feeder-dependent hiPSCs produced spheroids with two visibly partitioned domains when cultured in serum-containing medium (Fig. 1c, 2b). These particular spheroids were characterized by a SOX17+ endodermal periphery and an OCT4+ epithelial core. The SOX17+ cells were often mixed with an additional unidentified OCT4-/SOX17- population. These inner and outer tissue compartments were demarcated by a laminin-rich basal lamina (Fig. 1c). 
The SOX17+ endodermal cells were apparently responsible for ECM secretion, with disorganized aggregates of laminin visible in the spheroid outer layer (Fig. 1c). The underlying core of OCT4+ cells appeared as a radially arranged epithelium and expressed the basal lamina receptor aDG, which was enriched at the basal lamina

232 interface between the two tissue domains (Fig. 1c). Surprisingly, as little as one 233 passage on MEFs was sufficient to prime the hiPSCs for this self-organized 234 differentiation. Similar tissue patterning has been reported in spheroids derived from 235 mouse and human embryonic stem cells $[30,45]$. The observed structure is thought to 236 represent pre-gastrulation embryonic development, with an epiblast-like core and an 237 outer layer of extra-embryonic endoderm. Because the hiPSC-derived spheroids 238 produced with our protocol resemble this developmental stage, we refer to them 239 hereafter as embryoid bodies.

\section{Derivation of hiPSCs from Dystroglycanopathy Patients}

243 form of a basal lamina, we sought to apply this system for evaluating basal lamina 244 phenotypes in dystroglycanopathy - a diverse spectrum of muscular dystrophies often

245 co-morbid with brain malformation, characterized by ruptures in the basal lamina. We 246 reprogrammed hiPSCs from dermal fibroblasts of three unrelated individuals with a 247 genetic diagnosis of dystroglycanopathy. Each patient harbored predicted pathogenic 248 mutations in a different gene required for the glycosylation of aDG: $L A R G E, F K R P$, or POMT2 (Table 1). The LARGE patient has been reported in a previous publication [33]. 
Clinical presentation for all three patients included delayed motor milestones,

251

252

253

254

255

256

257

258

259

260

261

262

263

264

265

266

267

268

269

270

271

272

muscle weakness, and cognitive impairments (Table 1). Overall, the FKRP patient had

the mildest clinical and muscle biopsy findings, while the patient with the POMT2 mutations had the most severe findings. Brain magnetic resonance imaging showed minor white matter and structural abnormalities in the LARGE patient [33] and POMT2 patient (data not shown). Hypoglycosylation of aDG is the primary causative factor in the pathogenesis of dystroglycanopathy [34]. To evaluate the glycosylation status of aDG in patient cells, we used the IIH6C4 antibody that specifically recognizes the glycosylated form of $a D G$ (glyco-aDG). Consistent with clinical and genetic observations, we found reduced expression of glycosylated aDG in our patient hiPSCs (Fig. 3a). Two hiPSC clones were evaluated for each patient. All cell lines had a normal karyotype, and there were no discernible differences in the expression of pluripotent markers or propensity for germ layer differentiation (Fig. 3a, 4a-c).

To confirm the finding that patient cells express a hypoglycosylated form of aDG, we carried out western blots on hiPSC culture lysates using the IIH6C4 antibody. Probing for glycosylated aDG showed a reduction that roughly correlated with the clinical severity for each patient (Fig. 3b, c). Control hiPSCs expressed aDG glycoforms averaging $\sim 140 \mathrm{kDa}$, which is slightly less than reported in human muscle [26]. Previous analysis suggests that low molecular weight forms of aDG - a consequence of fewer glycan structures - is a specific biochemical hallmark associated with disease severity [14]. Our study followed this pattern, with cells from the clinically mild FKRP patient expressing glycosylated aDG of the same mass as controls but in reduced abundance. Glycosylated aDG from the LARGE patient, who was of intermediate severity, showed a 
$273 \sim 30 \mathrm{kDa}$ downward shift in molecular weight. hiPSCs from the severe POMT2 patient 274 were virtually devoid of glycosylated aDG.

275

To test the functional impact of these hypoglycosylated forms of aDG, we

276 performed a laminin overlay assay to measure the affinity of aDG for one of its ECM

277 ligands, laminin. All patient cell lines showed reduced aDG-laminin binding activity that

278 closely matched their degree of aDG hypoglycosylation (Fig. 3b, d). Blotting with 279 antibodies against the core peptide of aDG (core-aDG) and $\beta$-dystroglycan ( $\beta D G$ ) 280 indicated similar expression across all samples, demonstrating that the dystroglycan 281 proteins are expressed but that aDG is hypoglycosylated in dystroglycanopathy patient 282 hiPSCs (Fig. 3b).

\section{Differentiation of Embryoid Bodies from Dystroglycanopathy hiPSCs}

Muscle, eye, and brain abnormalities linked to basal lamina defects is a frequent exhibit the biochemical hallmark of the disease (i.e. hypoglycosylation of the basal lamina receptor $\mathrm{aDG}$ ), we next asked whether patient embryoid bodies can synthesize basal lamina. To investigate potential disease-related phenotypes, we initially restricted our analysis to embryoid bodies from the LARGE patient and POMT2 patient, who had moderate and severe clinical findings respectively.

We differentiated control and patient hiPSCs into embryoid bodies using the 293 protocol described earlier. Embryoid bodies from all cell lines contained a basal lamina 294 sandwiched by epithelial and endodermal compartments (Fig. 5a). There was 295 noticeable morphological variation across cell lines, possibly related to genetic 296 background or clonal differences. Particularly in embryoid bodies from the third control 
297 and the LARGE patient, there was an occasional inversion of tissue layers such that the 298 epithelial cells were on the exterior of the embryoid body (Fig. 5a).

Despite the heterogeneity between cultures, embryoid bodies from all control and

300

301

302

303

304

305

306

307

308

309

310

311

312

313

314

315

316

317

318

319 patient hiPSC clones were similarly capable of assembling a laminin-rich basal lamina at the surface of the OCT4+ epithelium. Additionally, all embryoid bodies showed the expected morphology of epithelial polarity. OCT4+ cells were radially arranged and exhibited apicobasal polarity, with F-actin distributed on the cellular edge opposite from the basal lamina (Fig. 5b). Thus, at this resolution of analysis, we detected no major phenotypic difference between control and dystroglycanopathy embryoid bodies.

Consistent with our finding in undifferentiated hiPSCs, LARGE and POMT2 embryoid bodies were minimally reactive to an antibody recognizing glycosylated aDG. However, all embryoid bodies expressed the other major basal lamina receptor, integrin- $\beta 1$ (Fig. 5c). Study of mouse embryoid bodies has shown functional redundancy between integrin- $\beta 1$ and $\alpha \mathrm{DG}$ in anchoring ECM molecules to the cell membrane [31]. This likely explains the ability of dystroglycanopathy patient embryoid bodies to assemble a basal lamina in the absence of aDG receptor function.

\section{Ultrastructural Basal Lamina Defects in Dystroglycanopathy Embryoid Bodies}

Previous studies of dystroglycanopathy patient and mouse tissue have revealed ultrastructural ECM defects $[14,25]$. To visualize embryoid body ECM ultrastructure, we employed transmission electron microscopy on thin sections of control and patient samples. In electron micrographs, basal lamina from control embryoid bodies was visible as a fibrous layer at the epithelial cell surface, roughly $100 \mathrm{~nm}$ thick (Fig. $5 \mathrm{~d}$ ). 
320 The overlying endodermal cell, some distance away, was always devoid of basal

321 lamina. The basal lamina was composed of a 'lamina lucida' and a 'lamina densa'

322 compartment. The lamina lucida - a thin electron-light layer at the epithelial plasma

323 membrane - is believed to be spanned by the laminin long arm bound to its cell surface

324 receptors, aDG and integrin [42]. The lamina densa - a thicker fibrous layer of electron-

325 dense material just above the lamina lucida - is comprised of laminin cross-linking 326 arms, perlecan, nidogen, and COLIV. However, the existence or extent of the lamina

327 lucida may also be an artifact of our sample dehydration method [6].

The epithelial cells of embryoid bodies showed typical features of lateral, apical,

329 and basal polarization. We observed electron-dense tight junctions at cell-cell borders,

330 and microvilli decorated the epithelial cells' apical aspect facing the embryoid body

331 lumen (Fig. 6). Nuclei were polarized toward the basal aspect of epithelial cells in

332 contact with the basal lamina (Fig. 5d). Occasionally, filamentous matrix could be seen

333 in the extracellular space between endodermal and epithelial cells, but it was rarely

334 attached to the basal lamina itself (Fig. 6). Therefore, the ECM structures in our

335 embryoid bodies meet the criteria for an epithelial basal lamina. However, they cannot

336 be categorized as a complete basement membrane, which requires an adjoined layer of

337 'lamina reticularis' fibrillar collagens [41].

339 due to a reduction of material in the lamina densa (Fig. 5d). Specifically, in POMT2 340 embryoid bodies, the basal lamina occasionally contained nanoscopic discontinuities

341 (Fig. 5d). The basal lamina in control embryoid bodies measured $105.8 \pm 6.1 \mathrm{~nm}$ thick. 
LARGE and POMT2 basal lamina were significantly thinner at $77.5 \pm 5.1$ and $69.1 \pm 4.5$ nm respectively (mean \pm s.e.m., $P=0.0022$ and $P<0.0001$ ) (Fig. 5e).

We considered whether a deficit of certain ECM molecules might explain the reduced thickness of patient basal lamina. In addition to laminin, aDG directly binds to perlecan, which in turn crosslinks nidogen and collagen type IV (COLIV) to form the basal lamina [39, 44]. Overall, there were no consistently detectable differences in the staining pattern or intensity of these basal lamina components between control and patient embryoid bodies (Fig. 7a). However, in some POMT2 embryoid bodies, there was occasionally a subtle loss of perlecan and COLIV co-localization with laminin (Fig. $7 b)$.

Over the course of differentiation, embryoid bodies were remarkably static in size, and there was no significant difference between control and POMT2 embryoid body surface area (Fig. 7c, d). This contrasts greatly with brain and muscle tissue, which undergo significant size expansion and mechanical strain during embryonic development and muscle contraction, respectively [20, 24]. Thus, our embryoid body differentiation protocol serves as a simplified model for patient-specific basal lamina assembly, without the additional variables of tissue movement and growth. In this system, dystroglycanopathy embryoid bodies with mutations in LARGE or POMT2 can synthesize a basal lamina of apparently typical molecular composition but with abnormal ultrastructure. These data corroborate published results demonstrating that aDG is generally dispensable for initial basal lamina formation [9, 30, 35].

Because our embryoid body differentiation protocol requires co-culture of hiPSCs with MEFs, we reasoned that abundant fibroblast ECM secretion could be masking 
disease-related deficits in basal lamina assembly. To evaluate this possibility, we labeled control and POMT2 hiPSCs and embryoid bodies with antibodies against human nuclear antigen (HuNu). In feeder-dependent hiPSC cultures, human hiPSCs were clearly distinguished by HuNu expression, while MEFs were heavily labeled by laminin antibodies (Fig. 8a). In embryoid bodies, hiPSCs and MEFs self-segregated within 24 hours of spheroid formation, with MEFs budding off and ultimately detaching from the differentiating embryoid body 2 - 4 days before basal lamina formation (Fig. 8b). Because all analyses were conducted on day 5 embryoid bodies, we believe the presence of MEFs is not a significant confounding factor in our experiments.

\section{Impaired Basal Lamina Assembly on Endoderm-Free Dystroglycanopathy} Embryoid Bodies

Knockout of dystroglycan in mouse embryonic stem cells and neural stem cells has been reported to reduce laminin polymerization at the cell surface, which is a prerequisite for basal lamina formation [21, 52]. Because we observed thinner basal lamina in dystroglycanopathy patient embryoid bodies, we next investigated whether this phenotype might be linked to a reduced ability to polymerize laminin. We developed an embryoid body culture protocol that both prevents the formation of laminin-secreting endoderm and obviates the need for MEF co-culture (Fig. 9a, b). This eliminated the major sources of ECM and allowed for precise control of laminin concentration by exogenous supplementation in the growth medium.

In the absence of laminin, endoderm-free embryoid bodies self-assembled into disorganized, cavitated spheroids (Fig. 9c). We supplemented various concentrations of 
388 laminin to control embryoid bodies and inspected the cultures after 48 hours. Amounts 389 greater than $100 \mu \mathrm{g} / \mathrm{mL}$ resulted in a thin layer of laminin at the surface of some 390 embryoid bodies. In embryoid bodies with significant laminin recruitment, the central 391 cavity was widened, and cells with direct laminin contact adopted a polarized orientation 392 (Fig. 9c). POMT2 embryoid bodies - from the most severe dystroglycanopathy patient 393 showed a striking absence of accumulated surface laminin except in the highest 394 concentration tested, $180 \mu \mathrm{g} / \mathrm{mL}$. To avoid ceiling and floor effects, we used $140 \mu \mathrm{g} / \mathrm{mL}$ 395 laminin in subsequent experiments, because approximately half of the control embryoid 396 body surface area bound laminin at this concentration (Fig. 9c). We tested endoderm-free embryoid bodies from control subjects and LARGE, 398 FKRP, and POMT2 dystroglycanopathy patients for their capacity to assemble laminin 399 (Fig. 10a). Compared to controls, which accumulated laminin on $65.7 \pm 6.3 \%$ of 400 surfaces, POMT2 embryoid bodies showed $20.5 \pm 5.2 \%$ surface laminin (mean \pm s.e.m., $401 \quad P=0.0006)$ (Fig. 10d). In contrast, LARGE and FKRP embryoid bodies assembled 48.4 $402 \pm 7.3 \%$ and $46.1 \pm 10 \%$ laminin respectively, which was also reduced but not 403 statistically different from controls $(P=0.405$ and $P=0.283)$ (Fig. 10d).

404

405 Ribitol Treatment Promotes Functional Glycosylation of aDG in FKRP Patient 406 Embryoid Bodies

FKRP is a glycosyltransferase enzyme that catalyzes the addition of ribitol 408 phosphate to $\mathrm{aDG}[13,27,40]$. This is an essential enzymatic step for the post409 translational installation of matriglycans on aDG, which are the structural basis for aDG- 
410 ligand binding [4]. Recently, dietary supplementation with ribitol was shown to improve

411 muscle phenotypes in a mouse model of FKRP-related dystroglycanopathy [5].

412

To determine the effect of ribitol on dystroglycanopathy patient hiPSCs, we

413 supplemented the culture medium with $3 \mathrm{mM}$ ribitol daily and harvested the cells 72

414 hours later. This dosage was based on previous reports of efficacious treatment with

$4153 \mathrm{mM}$ ribitol or CDP-ribitol in ISPD mutant dystroglycanopathy cell cultures [13, 27].

416 Ribitol treatment of FKRP hiPSCs greatly increased the abundance of glycosylated aDG

417 and resulted in a $20 \mathrm{kDa}$ upward shift in molecular weight (Fig. 10b, 11a). This change

418 was accompanied by a comparable increase in aDG-laminin binding. Ribitol-treated

419 LARGE hiPSCs were unchanged in glycosylated aDG quantity but showed a slight

420 increase in molecular weight (5-10 kDa). As would be expected, POMT2 hiPSCs, in

421 which glycosylated aDG is essentially absent, showed no improvement with ribitol

422 exposure (Fig. 10b). In addition to ribitol phosphate, FKRP is known to transfer glycerol

423 phosphate onto aDG, which inhibits its functional glycosylation. However, we found that

424 treating control and FKRP hiPSCs with $3 \mathrm{mM}$ glycerol daily for 72 hours had no

425 discernable effect on aDG glycosylation (Fig. 11b).

426

Because ribitol treatment resulted in a specific and profound improvement to

427 glycosylated aDG in FKRP hiPSCs, its effect was next assessed in FKRP endoderm-

428 free embryoid bodies. hiPSCs were treated with $3 \mathrm{mM}$ ribitol daily starting 72 hours

429 before embryoid body formation and continued throughout the experiment. Treatment of

430 FKRP embryoid bodies lead to a noticeable increase in the staining intensity of

431 glycosylated aDG (Fig. 10a, c). There was a slight but non-significant increase in

432 surface laminin polymerization on ribitol treated FKRP embryoid bodies $(62.1 \pm 6.6 \%)$ 
433 compared to untreated FKRP embryoid bodies $(46.1 \pm 10 \%$; mean \pm s.e.m, $P=0.566)$

434 (Fig. 10d). Importantly though, at laminin contact points, glycosylated aDG was

435 upregulated to the same level as controls (ribitol-treated FKRP: $107.2 \pm 3.4 \%$; untreated

436 FKRP: $44.5 \pm 3.5 \%$; normalized to control glyco-aDG, mean \pm s.e.m., $P<0.0001$ ) (Fig.

437 10e). These results demonstrate ribitol treatment as a viable therapeutic strategy for

438 upregulating aDG glycosylation in FKRP patient tissue. 


\section{Discussion}

442 vitro. We applied this method to study patients with dystroglycanopathy. We found that 443 patient hiPSC-derived embryoid bodies recapitulate the clinical disease spectrum and 444 exhibit ECM defects seen in animal models. Finally, this system allowed us to evaluate 445 the efficacy of ribitol supplementation - a recent candidate therapeutic for the dystroglycanopathies - in patient samples of different genotypes.

Embryoid body-based methods, which essentially involve culturing pluripotent stem cells in 3D aggregates, are widely used in stem cell research. Typical applications

449 include evaluating the pluripotency of new cell lines or as an intermediate stage during

450 differentiation toward specific lineages [28]. Under strict growth conditions, embryoid 451 bodies from mouse and human embryonic stem cells (ESCs) can exquisitely self452 organize into structures mimicking the pre-gastrulation embryo. These have served as 453 models for early embryonic events - epiblast polarization and ECM formation - that are 454 required for tissue genesis [29].

Our human hiPSC-derived embryoid bodies resemble their ESC-derived counterparts by similarly requiring serum-containing media and MEF co-culture to form

457 polarized epiblast, basal lamina, and extra-embryonic endoderm. It remains unclear why 458 these conditions uniquely enable such self-organization. BMPs, which are abundant in 459 serum [22], are known to induce extra-embryonic endoderm differentiation [16]. Co460 culture with MEFs can also influence the lineage bias of ESCs [32]. Thus, it may be a 461 combination of soluble factors from serum and MEFs that promotes the simultaneous 
462 existence of endoderm and epiblast cell populations in our hiPSC-derived embryoid 463 bodies.

Dual genetic deletion of $\mathrm{aDG}$ and integrin- $\beta 1$ prevents basement membrane 465 formation and epithelial polarization in mouse ESC-derived embryoid bodies. Because 466 these two receptors have overlapping roles, expression of one can partly rescue the 467 function of the other [31]. By studying dystroglycanopathy patient embryoid bodies, we 468 extend these findings to show that a moderate reduction in aDG receptor activity - with normal expression of both $\alpha \mathrm{DG}$ and integrin- $\beta 1$ core proteins - is sufficient to cause subtle ECM deficits that may underlie disease pathogenesis.

A thin and discontinuous basal lamina has been reported in the muscle of 472 dystroglycanopathy patients [25, 48], similar to our observation in patient embryoid 473 bodies. Also, the retina inner limiting basement membrane is thin, patchy, and less stiff 474 in Pomgnt1-null mice [23, 52]. One caveat of our hiPSC-derived embryoid body system 475 is that it seems to only recapitulate basal lamina development. It is unclear how the 476 observed basal lamina defects might translate to the formation of a full basement 477 membrane. Furthermore, the specific structural and molecular deficits underlying such 478 abnormally thin basal lamina are still unknown.

In contrast to the above findings, basement membranes in dystroglycan-null mouse embryoid bodies, and in the muscle of Large mutant mice and certain human 481 patients, are thicker than controls, sometimes with mislocalized ECM components [14, 482 30]. These dichotomous observations may be related to differences in ECM recruitment, 483 organization, and maintenance at the cell surface, as a consequence of reduced $\alpha D G$ 484 receptor activity but under specific tissue and disease state circumstances. One study 
485 reported a mixture of thinned and duplicated basal lamina in different regions of

486

487

488

489

490

491

492

493

494

495

496

497

498

499

500

501

502

503

504

505

506

507

dystroglycanopathy patient muscle [46]. Therefore, whether the diseased basal lamina

is thin and patchy, or thick and duplicated, may vary due to local tissue conditions.

The pathogenic mechanism of brain malformation in the dystroglycanopathies is still not fully understood. During brain development, the human cerebral cortex undergoes massive expansion and folding in the third trimester [12]. This rapid increase of surface area likely places mechanical strain on the brain's basement membrane casing, necessitating timely ECM remodeling to accommodate the larger area. In mice, genetic deletion of ECM genes or hypoglycosylation of $\alpha \mathrm{DG}$ both result in basement membrane rupture and ensuing tissue malformation during this most rapid phase of brain development $[2,19,37,43]$. These data present aDG as one of several laminin receptors that contributes to the efficient organization of ECM molecules into a coherent basement membrane.

Our result - that endoderm-free patient embryoid bodies show diminished laminin accumulation - corroborates evidence that $\alpha$ DG-deficient cells have reduced ECM-binding kinetics that might render basement membranes susceptible to mechanically-induced deformation [21, 52]. Crucially, we found that embryoid bodies undergo minimal volumetric growth. This could explain the relatively mild phenotypes in embryoid bodies of even the most severe dystroglycanopathy patient.

The human brain specifically undergoes greatest expansion in the occipital, temporal, and lateral parietal cortices [12]. Such regional growth dynamics suggest one possible basis for the spatial arrangement of the typical cortical malformation in the dystroglycanopathies, which are fundamentally due to breaches in basement membrane 
508 integrity, resulting in cellular over-migration beyond the confines of the $[1,7,33,50]$.

509 These particular growth dynamics may also underlie some of the differences between

510 human patients and mouse models [3].

511 There is currently no effective treatment for the dystroglycanopathies, and the

512 diversity of underlying genetic causes for the disease presents a challenge for targeted

513 therapies. Supplementation of the sugar alcohol ribitol has recently emerged as a 514 promising therapeutic for specific classes of dystroglycanopathy. In mammalian cells,

515 the enzyme ISPD synthesizes CDP-ribitol from ribitol, which is then attached to aDG by

516 the glycosyltransferases FKTN and FKRP [13, 27, 40]. This enzymatic process is a 517 critical step in constructing the laminin-binding glycan of aDG.

518 Treatment of ISPD mutant cells with ribitol or CDP-ribitol promotes aDG 519 glycosylation [13, 27], and dietary ribitol supplementation rescues muscle phenotypes in 520 Fkrp mutant mice [5]. Here, we extend this concept from animal models to a human 521 patient-derived system. We found specific efficacy of ribitol on FKRP patient hiPSCs, as

522 evidenced by a complete rescue of glycosylated laminin-binding aDG. There was 523 minimal effect on LARGE and no effect on POMT2 patient hiPSCs, as would be 524 expected from the location of these genetic forms in the pathway of aDG glycosylation.

525 In FKRP endoderm-free embryoid bodies, ribitol significantly upregulated glycosylated $526 \mathrm{aDG}$ at the laminin interface. There was also slightly increased accumulation of laminin 527 at the embryoid body surface, but this in vitro system may lack the complexity or 528 sensitivity to further detect functional improvements in an already relatively mild patient.

529 The FKRP patient in this study harbors a L276I mutation, the most common 530 variant in the FKRP-related dystroglycanopathies [11]. We speculate that ribitol 
531 supplementation may be a rational and effective treatment, in particular for mild-to-

532 moderate FKRP and FKTN patients with residual ribitol transferase function that can be

533 boosted by the additional supply of substrate. It remains to be investigated whether

534 ribitol would also benefit additional groups of dystroglycanopathy patients. Collectively,

535 these data establish a system to interrogate basal lamina structure and ECM receptor

536 function in patient tissue, expanding the options for personalized phenotyping and drug

537 evaluation in the dystroglycanopathies. 


\section{Acknowledgements}

539 We would like to thank the patients and their families for their participation. We are

540 grateful to Susan Cheng, Virginia Crocker, and Sandra Lara for their EM technical

541 support. Electron microscopy was performed in the NINDS EM facility. This work was

542 funded by the US National Institutes of Health Intramural Research Program in the

543 National Institute of Neurological Disorders and Stroke.

544

545 Conflict of interest

546 The authors declare that they have no competing interests.

547

548 Author contributions

549 ARN, KZ, and CGB conceived and designed the study. ARN performed all experiments.

550 MML contributed to data collection and image analyses. BSM assisted in developing the

551 embryoid body protocol. ARN wrote the manuscript and all authors edited the 552 manuscript. 
554

555

556

557

558

559

560

561

562

563

564

565

566

567

568

569

570

571

572

573

574

575

576

577

578

579

580

581

582

583

584

585

586

587

588

589

590

\section{References}

1. Aida N, Tamagawa K, Takada K, Yagishita A, Kobayashi N, Chikumaru K, Iwamoto H (1996) Brain MR in Fukuyama congenital muscular dystrophy. Am J Neuroradiol 17:605-13

2. Booler HS, Pagalday-Vergara V, Williams JL, Hopkinson M, Brown SC (2016) Evidence of early defects in Cajal Retzius cell localisation during brain development in a mouse model of dystroglycanopathy. Neuropathol Appl Neurobiol 43:330-345. doi: 10.1111/ijlh.12426

3. Booler HS, Williams JL, Hopkinson M, Brown SC (2015) Degree of Cajal-Retzius cell mislocalisation correlates with the severity of structural brain defects in mouse models of dystroglycanopathy. Brain Pathol 26:465-478. doi: 10.1111/bpa.12306

4. Briggs D., Strazzulli A, Moracci M, Yu L, Yoshida-Moriguchi T, Zheng T, Venzke D, Anderson M, Hohenester E, Campbell KP (2016) Structural basis of laminin binding to the LARGE glycans on dystroglycan. Nat Chem Biol 12:810-814. doi: 10.2210/PDB5IK7/PDB

5. Cataldi MP, Lu P, Blaeser A, Lu QL (2018) Ribitol restores functionally glycosylated $\alpha$-dystroglycan and improves muscle function in dystrophic FKRPmutant mice. Nat Commun 9:3448. doi: 10.1038/s41467-018-05990-z

6. Chan FL, Inoue S (1994) Lamina lucida of basement membrane: An artefact. Microsc Res Tech 28:48-59. doi: 10.1002/jemt.1070280106

7. Clement E, Mercuri E, Godfrey C, Smith J, Robb S, Kinali M, Straub V, Bushby K, Manzur A, Talim B, Cowan F, Quinlivan R, Klein A, Longman C, McWilliam R, Topaloglu H, Mein R, Abbs S, North K, Barkovich AJ, Rutherford M, Muntoni F (2008) Brain involvement in muscular dystrophies with defective dystroglycan glycosylation. Ann Neurol 64:573-582. doi: 10.1002/ana.21482

8. Clement EM, Feng L, Mein R, Sewry CA, Robb SA, Manzur AY, Mercuri E, Godfrey C, Cullup T, Abbs S, Muntoni F (2012) Relative frequency of congenital muscular dystrophy subtypes: Analysis of the UK diagnostic service 2001-2008. Neuromuscul Disord 22:522-527. doi: 10.1016/j.nmd.2012.01.010

9. Cote PD, Moukhles H, Lindenbaum M, Carbonetto S (1999) Chimaeric mice deficient in dystroglycans develop muscular dystrophy and have disrupted myoneural synapses. Nat Genet 23:338-342. doi: 10.1038/15519

10. Devisme L, Bouchet $C$, Gonzalès $M$, Alanio $E$, Bazin A, Bessières B, Bigi N, Blanchet $P$, Bonneau $D$, Bonnières $M$, Bucourt $M$, Carles $D$, Clarisse $B$, Delahaye S, Fallet-Bianco C, Figarella-Branger D, Gaillard D, Gasser B, Delezoide A-L, Guimiot $F$, Joubert $M$, Laurent $N$, Laquerrière A, Liprandi A, Loget $P$, Marcorelles P, Martinovic J, Menez F, Patrier S, Pelluard F, Perez M-J, Rouleau C, Triau S, 
Attié-Bitach T, Vuillaumier-Barrot S, Seta N, Encha-Razavi F (2012) Cobblestone lissencephaly: neuropathological subtypes and correlations with genes of dystroglycanopathies. Brain 135:469-482. doi: 10.1093/brain/awr357

11. Frosk P, Greenberg CR, Tennese AAP, Lamont R, Nylen E, Hirst C, Frappier D, Roslin NM, Zaik M, Bushby K, Straub V, Zatz M, De Paula F, Morgan K, Fujiwara TM, Wrogemann K (2005) The most common mutation in FKRP causing limb girdle muscular dystrophy type 21 (LGMD2I) may have occurred only once and is present in Hutterites and other populations. Hum Mutat 25:38-44. doi: 10.1002/humu.20110

12. Garcia KE, Robinson EC, Alexopoulos D, Dierker DL, Glasser MF, Coalson TS, Ortinau CM, Rueckert D, Taber LA, Van Essen DC, Rogers CE, Smyser CD, Bayly P V. (2018) Dynamic patterns of cortical expansion during folding of the preterm human brain. Proc Natl Acad Sci 115:3156-3161. doi: 10.1073/pnas. 1715451115

13. Gerin I, Ury B, Breloy I, Bouchet-Seraphin C, Bolsée J, Halbout M, Graff J, Vertommen D, Muccioli GG, Seta N, Cuisset J-M, Dabaj I, Quijano-Roy S, Grahn A, Van Schaftingen E, Bommer GT (2016) ISPD produces CDP-ribitol used by FKTN and FKRP to transfer ribitol phosphate onto a-dystroglycan. Nat Commun 7:11534. doi: $10.1038 /$ ncomms 11534

14. Goddeeris MM, Wu B, Venzke D, Yoshida-Moriguchi T, Saito F, Matsumura K, Moore SA, Campbell KP (2013) LARGE glycans on dystroglycan function as a tunable matrix scaffold to prevent dystrophy. Nature 503:136-140. doi: $10.1038 /$ nature12605

15. Godfrey C, Foley AR, Clement E, Muntoni F (2011) Dystroglycanopathies: coming into focus. Curr Opin Genet Dev 21:278-285. doi: 10.1016/j.gde.2011.02.001

16. Graham SJL, Wicher KB, Jedrusik A, Guo G, Herath W, Robson P, ZernickaGoetz M (2014) BMP signalling regulates the pre-implantation development of extra-embryonic cell lineages in the mouse embryo. Nat Commun 5:5667. doi: $10.1038 /$ ncomms 6667

17. Graziano A, Messina S, Bruno C, Pegoraro E, Magri F (2015) Prevalence of congenital muscular dystrophy in Italy: A population study. Neurology 84:904911. doi: $10.1212 /$ WNL.0000000000001303

18. Grunseich C, Zukosky K, Kats IR, Ghosh L, Harmison GG, Bott LC, Rinaldi C, Chen K, Chen G, Boehm M, Fischbeck KH (2014) Stem cell-derived motor neurons from spinal and bulbar muscular atrophy patients. Neurobiol Dis 70:1220. doi: 10.1016/j.nbd.2014.05.038

19. Halfter W, Dong S, Yip Y-P, Willem M, Mayer U (2002) A critical function of the pial basement membrane in cortical histogenesis. J Neurosci 22:6029-6040. doi: 20026580 
20. Han R, Kanagawa M, Yoshida-Moriguchi T, Rader EP, Ng RA, Michele DE, Muirhead DE, Kunz S, Moore SA, lannaccone ST, Miyake K, McNeil PL, Mayer U, Oldstone MBA, Faulkner JA, Campbell KP (2009) Basal lamina strengthens cell membrane integrity via the laminin $\mathrm{G}$ domain-binding motif of a-dystroglycan. Proc Natl Acad Sci 106:12573-12579. doi: 10.1073/pnas.0906545106

21. Henry MD, Campbell KP (1998) A Role for Dystroglycan in Basement Membrane Assembly. Cell 95:859-870. doi: 10.1016/S0092-8674(00)81708-0

22. Herrera B, Inman GJ (2009) A rapid and sensitive bioassay for the simultaneous measurement of multiple bone morphogenetic proteins. Identification and quantification of BMP4, BMP6 and BMP9 in bovine and human serum. BMC Cell Biol 10:20. doi: 10.1186/1471-2121-10-20

23. Hu H, Candiello J, Zhang P, Ball SL, Cameron D a, Halfter W (2010) Retinal ectopias and mechanically weakened basement membrane in a mouse model of muscle-eye-brain (MEB) disease congenital muscular dystrophy. Mol Vis $16: 1415-1428$

24. Hu H, Yang Y, Eade A, Xiong Y, Qi Y (2007) Breaches of the Pial Basement Membrane and Disappearance of the Glia Limitans During Development Underlie the Cortical Lamination Defect in the Mouse Model of Muscle-Eye-Brain Disease. J Comp Neurol 502:168-183. doi: 10.1002/cne

25. Ishii H, Hayashi YK, Nonaka I, Arahata K (1997) Electron microscopic examination of basal lamina in Fukuyama congenital muscular dystrophy. Neuromuscul Disord 7:191-7

26. Jensen BS, Willer T, Saade DN, Cox MO, Mozaffar T, Scavina M, Stefans VA, Winder TL, Campbell KP, Steven A, Mathews KD (2015) GMPPB-Associated Dystroglycanopathy: Emerging Common Variants with Phenotype Correlation. Hum Mutat. doi: 10.1002/humu.22898

27. Kanagawa M, Kobayashi K, Tajiri M, Manya H, Kuga A, Yamaguchi Y (2016) Identification of a Post-translational Modification with Ribitol-Phosphate and Its Defect in Muscular Dystrophy. Cell Rep 14:2209-2223. doi: 10.1016/j.celrep.2016.02.017

28. Kurosawa $\mathrm{H}$ (2007) Methods for inducing embryoid body formation: in vitro differentiation system of embryonic stem cells. J Biosci Bioeng 103:389-398. doi: 10.1263/jbb.103.389

29. Li S, Edgar D, Fässler R, Wadsworth W, Yurchenco PD (2003) The role of laminin in embryonic cell polarization and tissue organization. Dev Cell 4:613-624. doi: 10.1016/S1534-5807(03)00128-X

30. Li S, Harrison D, Carbonetto S, Fässler R, Smyth N, Edgar D, Yurchenco PD (2002) Matrix assembly, regulation, and survival functions of laminin and its receptors in embryonic stem cell differentiation. J Cell Biol 157:1279-1290. doi: 
670

671

672

673

674

675

676

677

678

679

680

681

682

683

684

685

686

687

688

689

690

691

692

693

694

695

696

697

698

699

700

701

702

703

704

705

706

707

31. Li S, Qi Y, McKee K, Liu J, Hsu J, Yurchenco PD (2017) Integrin and dystroglycan compensate each other to mediate laminin-dependent basement membrane assembly and epiblast polarization. Matrix Biol 57:272-284. doi:

10.1016/j.matbio.2016.07.005

32. Lippmann ES, Estevez-Silva MC, Ashton RS (2014) Defined human pluripotent stem cell culture enables highly efficient neuroepithelium derivation without small molecule inhibitors. Stem Cells 32:1032-1042. doi: 10.1002/stem.1622

33. Meilleur KG, Zukosky K, Medne L, Fequiere P, Powell-Hamilton N, Winder TL, Alsaman A, El-Hattab AW, Dastgir J, Hu Y, Donkervoort S, Golden JA, Eagle R, Finkel R, Scavina M, Hood IC, Rorke-Adams LB, Bönnemann CG (2014) Clinical, Pathologic, and Mutational Spectrum of Dystroglycanopathy Caused by LARGE Mutations. J Neuropathol Exp Neurol 73:425-441

34. Michele DE, Barresi R, Kanagawa M, Saito F, Cohn RD, Satz JS, Dollar J, Nishino I, Kelley RI, Somer H, Straub V, Mathews KD, Moore SA, Campbell KP (2002) Post-translational disruption of dystroglycan-ligand interactions in congenital muscular dystrophies. Nature 418:417-421. doi: 10.1038/nature00837

35. Moore SA, Saito F, Chen J, Michele DE, Henry MD, Messing A, Cohn RD, RossBarta SE, Westra S, Williamson RA, Hoshi T, Campbell KP (2002) Deletion of brain dystroglycan recapitulates aspects of congenital muscular dystrophy. Nature 418:422-425. doi: 10.1038/nature00838

36. Morrissey MA, Sherwood DR (2015) An active role for basement membrane assembly and modification in tissue sculpting. J Cell Sci 128:1661-1668. doi: 10.1242/jcs. 168021

37. Nakagawa N, Yagi H, Kato K, Takematsu H, Oka S (2015) Ectopic clustering of Cajal-Retzius and subplate cells is an initial pathological feature in Pomgnt2knockout mice, a model of dystroglycanopathy. Sci Rep 5:11163. doi: 10.1038/srep11163

38. Nickolls AR, Bönnemann CG (2018) The roles of dystroglycan in the nervous system: insights from animal models of muscular dystrophy. Dis Model Mech 11:dmm035931. doi: 10.1242/dmm.035931

39. Peng HB, Ali A, Daggett DF, Rauvala H, Hassell JR, Smalheiser NR (1998) The relationship between perlecan and dystroglycan and its implication in the formation of the neuromuscular junction. Cell Adhes Commun 5:475-489. doi: $10.3109 / 15419069809005605$

40. Praissman JL, Willer T, Sheikh MO, Toi A, Chitayat D, Lin Y-Y, Lee H, Stalnaker SH, Wang S, Prabhakar PK, Nelson SF, Stemple DL, Moore SA, Moremen KW, Campbell KP, Wells $L$ (2016) The functional O-mannose glycan on alphadystroglycan contains a phospho-ribitol primed for matriglycan addition. Elife 
5:e14473. doi: 10.7554/eLife.14473

41. Sanes JR (1982) Laminin, fibronectin, and collagen in synaptic and extrasynaptic portions of muscle fiber basement membrane. J Cell Biol 93:442-451. doi: 10.1083/jcb.93.2.442

42. Schittny JC, Timpl R, Engel J (1988) High resolution immunoelectron microscopic localization of functional domains of laminin, nidogen, and heparan sulfate proteoglycan in epithelial basement membrane of mouse cornea reveals different topological orientations. J Cell Biol 107:1599-1610. doi: 10.1083/jcb.107.4.1599

43. Sudo A, Kanagawa M, Kondo M, Chiyomi I, Kobayashi K, Endo M, Minami Y, Aiba A, Toda T (2018) Temporal requirement of dystroglycan glycosylation during brain development and rescue of severe cortical dysplasia via gene delivery in the fetal stage. Hum Mol Genet 27:1174-1185. doi: 10.1093/hmg/ddx143

44. Talts JF, Andac Z, Göhring W, Brancaccio A, Timpl R (1999) Binding of the G domains of laminin $\alpha 1$ and $\alpha 2$ chains and perlecan to heparin, sulfatides, $\alpha-$ dystroglycan and several extracellular matrix proteins. EMBO J 18:863-870. doi: 10.1093/emboj/18.4.863

45. Ungrin MD, Joshi C, Nica A, Bauwens C, Zandstra PW (2008) Reproducible, ultra high-throughput formation of multicellular organization from single cell suspension-derived human embryonic stem cell aggregates. PLoS One 3. doi: 10.1371/journal.pone.0001565

46. Vajsar J, Ackerley C, Chitayat D, Becker LE (2000) Basal lamina abnormality in the skeletal muscle of Walker-Warburg syndrome. Pediatr Neurol 22:139-143. doi: 10.1016/S0887-8994(99)00129-0

47. Winograd-Katz SE, Fässler R, Geiger B, Legate KR (2014) The integrin adhesome: From genes and proteins to human disease. Nat Rev Mol Cell Biol 15:273-288. doi: 10.1038/nrm3769

48. Yamamoto T, Toyoda C, Kobayashi M, Kondo E, Saito K, Osawa M (1997) Pialglial barrier abnormalities in fetuses with Fukuyama congenital muscular dystrophy. Brain Dev 19:35-42. doi: 10.1016/s0387-7604(96)00056-3

49. Yoshida-Moriguchi T, Campbell KP (2015) Matriglycan: a novel polysaccharide that links dystroglycan to the basement membrane. Glycobiology 25:702-713. doi: 10.1093/glycob/cwv021

50. Yoshioka M, Kobayashi K, Toda T (2017) Novel FKRP mutations in a Japanese MDC1C sibship clinically diagnosed with Fukuyama congenital muscular dystrophy. Brain Dev 39:869-872. doi: 10.1016/j.braindev.2017.05.013

51. Yurchenco PD (2011) Basement Membranes: Cell Scaffoldings and Signaling Platforms. Cold Spring Harb Perspect Biol 3:a004911. doi:

10.1101/cshperspect.a004911 
746 52. Zhang P, Yang Y, Candiello J, Thorn TL, Gray N, Halfter WM, Hu H (2013) $747 \quad$ Biochemical and biophysical changes underlie the mechanisms of basement membrane disruptions in a mouse model of dystroglycanopathy. Matrix Biol 32:196-207. doi: 10.1016/j.matbio.2013.02.002 


\section{Tables}

752

\section{Table 1}

754 Characteristics of study subjects

\begin{tabular}{|c|c|c|c|c|c|}
\hline Subject & Age & Gender & Clinical presentation & Diagnosis & Genotype \\
\hline Control-1 & Neonate & Male & $\mathrm{N} / \mathrm{A}$ & $\mathrm{N} / \mathrm{A}$ & $\mathrm{N} / \mathrm{A}$ \\
\hline Control-2 & Neonate & Male & $\mathrm{N} / \mathrm{A}$ & $\mathrm{N} / \mathrm{A}$ & $\mathrm{N} / \mathrm{A}$ \\
\hline Control-3 & 51 & Female & $\mathrm{N} / \mathrm{A}$ & $\mathrm{N} / \mathrm{A}$ & $\mathrm{N} / \mathrm{A}$ \\
\hline LARGE & 6 & Female & $\begin{array}{l}\text { Delayed motor } \\
\text { milestones, weakness } \\
\text { and general hypotonia, } \\
\text { able to walk stairs, mild } \\
\text { autistic behavior, mild } \\
\text { muscle biopsy, } \\
\text { lissencephaly on brain } \\
\text { MRI }\end{array}$ & $\begin{array}{l}\text { Congenital } \\
\text { muscular } \\
\text { dystrophy } \\
\text { type 1D }\end{array}$ & $\begin{array}{l}\text { LARGE1: } \\
\text { Heterozygous } \\
\text { - Exon } 7 \text { deletion } \\
\text { - Exons } 3-7 \text { deletion }\end{array}$ \\
\hline FKRP & 3 & Male & $\begin{array}{l}\text { Delayed motor } \\
\text { milestones, mild } \\
\text { weakness, trunk } \\
\text { hypotonia, waddling gait, } \\
\text { able to run, mild autistic } \\
\text { behavior, moderate } \\
\text { dystrophy on muscle } \\
\text { biopsy, normal basement } \\
\text { membrane on TEM }\end{array}$ & $\begin{array}{l}\text { Early onset } \\
\text { limb-girdle } \\
\text { muscular } \\
\text { dystrophy } \\
\text { type 2l }\end{array}$ & $\begin{array}{l}\text { FKRP: Heterozygous } \\
\text { - c.C826A (p.L276I) } \\
\text { - c.G534T (p.W178C) }\end{array}$ \\
\hline POMT2 & $41 / 2$ & Female & $\begin{array}{l}\text { Severely delayed motor } \\
\text { milestones, hip dysplasia } \\
\text { at birth, weakness and } \\
\text { general hypotonia, } \\
\text { unable to stand, joint } \\
\text { contractures, failure to } \\
\text { thrive, delayed speech } \\
\text { acquisition, white matter } \\
\text { hyperintensities on MRI }\end{array}$ & $\begin{array}{l}\text { Muscle-Eye- } \\
\text { Brain } \\
\text { Disease }\end{array}$ & $\begin{array}{l}\text { POMT2: Homozygous } \\
\text { - c.G1057A (p.G353S) }\end{array}$ \\
\hline
\end{tabular}


756 Table 2

757 Antibodies for immunofluorescence and western blots

\begin{tabular}{|c|c|c|c|}
\hline Antibody & Dilution & Company & Catalog Number \\
\hline sox17 & $1: 100$ & Abcam & ab84990 \\
\hline OCT4 & $1: 200$ & Abcam & $a b 134218$ \\
\hline Laminin & $\begin{array}{l}1: 1,000 \text { (IF) } \\
1: 5,000 \text { (WB) }\end{array}$ & Sigma-Aldrich & L9393 \\
\hline $\begin{array}{l}\text { Glyco- } a \text { DG } \\
\text { (IIH6C4) }\end{array}$ & $\begin{array}{l}1: 200 \text { (IF) } \\
1: 1,000 \text { (WB) }\end{array}$ & Millipore & $05-593$ \\
\hline Core-aDG & $1: 500$ & R\&D Systems & AF6868 \\
\hline$\beta D G$ & $1: 10,000$ & GeneTex & GTX124225 \\
\hline Nestin & $1: 500$ & Millipore & ABD69 \\
\hline SSEA3 & $1: 200$ & Abcam & ab16286 \\
\hline SSEA4 & $1: 500$ & STEMCELL Technologies & 60062 \\
\hline $\begin{array}{l}\text { F-actin } \\
\text { (Phalloidin-647) }\end{array}$ & $1: 100$ & Thermo Fisher & A22287 \\
\hline Perlecan & $1: 100$ & Millipore & MAB1948-P \\
\hline Nidogen & $1: 50$ & $R \& D$ Systems & MAB2570 \\
\hline COLIV & $1: 1,000$ & Chemicon & MAB1430 \\
\hline $\mathrm{HuNu}$ & $1: 100$ & Millipore & MAB1281 \\
\hline
\end{tabular}

758

$759 \mathrm{IF}$, immunofluorescence; WB, western blot 
760 Figure legends

761

762 Fig. 1

763 Self-Organization of Basal Lamina-Containing Embryoid Bodies from Human 764 hiPSCs.

765 (a) Schematic of embryoid body differentiation protocol from feeder-dependent hiPSCs. 766 X-VIVO refers to X-VIVO 10 medium (see experimental procedures). (b) Phase-contrast 767 representation of embryoid body differentiation. hiPSCs were seeded on day 0 in a 768 microwell plate to form spheroids of roughly 2,000 cells on day 1 . The spheroids were 769 then maintained in suspension culture until day 5. Scale bars, $500 \mu \mathrm{m}$. (c) Phase770 contrast and immunohistochemistry on day 5 embryoid bodies showing two distinct 771 tissue domains, with a basal lamina in contact with the interior OCT4+ cells expressing 772 glycosylated aDG (glyco-aDG). Scale bars, $50 \mu \mathrm{m}$.

773

$774 \quad$ Fig. 2

775 Culture Conditions Impact the Lineage Outcome of Human hiPSC-Derived 776 Embryoid Bodies

777 (a) Phase-contrast and immunohistochemistry of hiPSC spheroids maintained in $20 \%$ 778 serum-replacement medium for 5 days. Feeder-dependent spheroids were derived from 779 hiPSCs cultured on a feeder layer of MEFs for at least one passage. (b) Phase-contrast 780 and immunohistochemistry of day 5 spheroids in serum-containing medium. Phase781 contrast scale bars, $500 \mu \mathrm{m}$; fluorescence scale bars, $50 \mu \mathrm{m}$. 
784

785

786

787

788

789

790

791

792

793

794

795

796

797

798

799

800

801

802

803

804

805

806 Fig. 5

\section{Dystroglycanopathy Patient hiPSCs Express Hypoglycosylated Forms of aDG}

(a) Immunocytochemistry and karyotype analyses of control and dystroglycanopathy patient-derived hiPSCs. Scale bars, $200 \mu \mathrm{m}$. (b) Western blots on hiPSC protein lysates. $\beta D G$ was used as a loading control. The asterisk indicates the molecular weight of endogenous laminin in the samples. Each lane represents one cell line (for controls) or one clone (for patients). (c, d) Quantification of western blots on glycosylated aDG and the laminin overlay assay. Band intensity for each sample was normalized to $\beta D G$, and all samples are expressed as a percent of control. Three control cell lines and two clones per patient were used, $\mathrm{n}=3$ technical replicates per clone. Values expressed as mean \pm s.e.m. Post-hoc comparisons ${ }^{*} P<0.05,{ }^{* *} P<0.01,{ }^{* * *} P<0.0001$.

Fig. 4

hiPSCs from Dystroglycanopathy Patients Show Normal Germ Layer Differentiation

(a) Immunocytochemistry of ectoderm markers. hiPSCs were differentiated via dual SMAD inhibition by treating with LDN-193189 and SB431542 for 6 days. (b) Mesoderm differentiation was mediated by Activin $A$ and Wnt3a treatment. TBX1 immunocytochemistry was performed after 2 days and GATA4 after 3 days. (c) Endoderm differentiation was also induced by Activin $A$ and Wnt3a. Immunocytochemistry was performed after 3 days. hESC, H9 human embryonic stem cell line. Scale bars, $100 \mu \mathrm{m}$. 
(a-c) Representative immunohistochemistry images of control and patient embryoid bodies at day 5 of differentiation. At least three independent differentiations were carried

811 out on each of three control hiPSC lines and two hiPSC clones from the LARGE and

812 POMT2 patient. Scale bars, $50 \mu \mathrm{m}$. (d) Transmission electron micrographs of embryoid

813 body basal lamina. Asterisk, nucleus; arrows, basal lamina; arrowheads, plasma

814 membrane; scale bars, $500 \mu \mathrm{m}$. (e) Measurements of basal lamina thickness. Three

815 control lines and two clones each from the LARGE and POMT2 patients were used. For

816 each clone, micrographs of $n \geq 10$ different basal lamina regions were collected across

$817 \geq 5$ embryoid bodies from 2-3 independent differentiations. Values displayed as mean \pm

s.e.m. Post-hoc comparisons ${ }^{* *} \mathrm{P}<0.01,{ }^{* * *} \mathrm{P}<0.0001$.

Fig. 6

\section{Ultrastructural Examination of Embryoid Body Morphology}

822 Representative transmission electron micrographs depicting the ultrastructural 823 morphology of embryoid body epithelium. Rare attachment of fibrillar matrix to the basal 824 lamina indicated by asterisk. Arrows demonstrate epithelial tight junctions, and the 825 arrow head indicates apical microvilli. Scale bar, $500 \mathrm{~nm}$.

826

827 Fig. 7

828 Analysis of Basal Lamina Components and Growth Characteristics of 829 Dystroglycanopathy Embryoid Bodies 
830 (a) Representative antibody labeling to assess co-localization of laminin with other basal 831 lamina constituents. Embryoid bodies from three controls and two clones per patient

832 were used. Scale bar, $50 \mu \mathrm{m}$. (b) Examples of occasionally separate localization of 833 laminin, perlecan, and COLIV in POMT2 embryoid bodies. The arrow shows localization 834 of perlecan outside the basal lamina. Arrowheads indicate the presence of laminin 835 without perlecan or COLIV. Scale bar, $50 \mu \mathrm{m}$. (c) Phase-contrast images of embryoid 836 body differentiation on days 1, 3, and 5. Scale bar, $100 \mu \mathrm{m}$. (d) Quantification of

837 embryoid body size over time based on averaged cross-sectional area measurements 838 in phase-contrast images from $n=3$ differentiations. At least 75 embryoid bodies per 839 line were analyzed during each differentiation. No statistical significance was found 840 between control and patient at any time point $(P>0.05)$, values expressed as mean \pm 841 s.d.

842

$843 \quad$ Fig. 8

844 Morphologically Mature Embryoid Bodies are Virtually Devoid of MEFs 845 (a) Immunocytochemistry of feeder-dependent hiPSCs to distinguish human cells 846 (HuNu) from MEFs. Control-1 hiPSCs and one hiPSC clone of the POMT2 patient were 847 used. (b) Embryoid bodies at different time points, derived from control and POMT1 848 hiPSCs. Scale bars, $100 \mu \mathrm{m}$.

849

$850 \quad$ Fig. 9

851 Assembly of Exogenous Laminin on Endoderm-Free Embryoid Bodies 
852 (a, b) Schematic and phase-contrast of endoderm-free embryoid body culture protocol.

853 Feeder-free hiPSCs were seeded on day 0 in microwell plates to form spheroids of

854 roughly 1,000 cells by day 1 . The spheroids were transferred to suspension culture

855 supplemented with laminin for 48 hours. (c) Immunohistochemistry demonstrating the

856 effect of increasing laminin concentration on endoderm-free embryoid bodies. Embryoid

857 bodies were supplemented with varying concentrations of laminin on day 1 and 858 collected for analysis 48 hours later. Scale bars, $100 \mu \mathrm{m}$.

859

860 Fig. 10

861

Patient-Specific Differences in Laminin Assembly and Response to Ribitol

862 Treatment

863 (a) Immunohistochemistry on day 3 endoderm-free embryoid bodies. Scale bar, 200

$864 \mu \mathrm{m}$. (b) Western blotting of protein lysates from hiPSC cultures. hiPSCs were 865 supplemented with daily medium changes with (+) or without (-) $3 \mathrm{mM}$ ribitol for 72

866 hours before protein was collected. Asterisk indicates the position of endogenous

867 laminin. (c) Immunohistochemistry on day 3 endoderm-free embryoid bodies to assess

868 the effect of ribitol treatment on the localization and glycosylation of aDG in the FKRP

869 patient. (d) Quantification of the percent embryoid body surface area covered by

870 laminin. Three controls and two clones per patient were analyzed across $n=3$

871 differentiations each. Values plotted as mean \pm s.e.m. Post-hoc comparisons ** $\mathrm{P}<$

$8720.01,{ }^{* * *} \mathrm{P}<0.001$. (e) Quantification of glycosylated aDG staining intensity at the

873 embryoid body surface. Three controls and two FKRP clones with and without ribitol

874 were analyzed and normalized to a percentage of the controls. For each clone, $n=27$ 
875 surface regions of $50 \mu \mathrm{m}$ length were quantified across three differentiations. Values

876 graphed as mean \pm s.e.m. Post-hoc comparisons ${ }^{* \star * *} P<0.0001$.

877

878 Fig. 11

879 Ribitol Treatment Promotes Functional Glycosylation of aDG in FKRP hiPSCs

880 (a) Additional western blots on control and FKRP patient hiPSCs. Cells were 881 supplemented with $3 \mathrm{mM}$ ribitol in daily medium changes for 72 hours before protein

882 was harvested. Asterisks indicate position of endogenous laminin in the samples. 883 Lipofectamine (Lipo.) (STEM00003, Thermo Fisher) was tested in conjunction with 884 ribitol treatment to enhance delivery to cells. In this condition, ribitol and lipofectamine 885 were administered for only 24 hours following manufacturer's instructions, and the cells 886 were collected 72 hours later. No apparent difference was observed between 24-hour 887 lipofectamine-delivered and 72 hour free-uptake of ribitol. (b) Daily administration of 3 $888 \mathrm{mM}$ glycerol for 72 hours shows no effect on aDG by western blot. 
a

Days

Culture iPSCs on MEFs Suspension culture

Procedures

Spheroid formation

Refresh medium

Medium

$20 \%$ KSR

X-VIVO

$20 \%$ serum

b
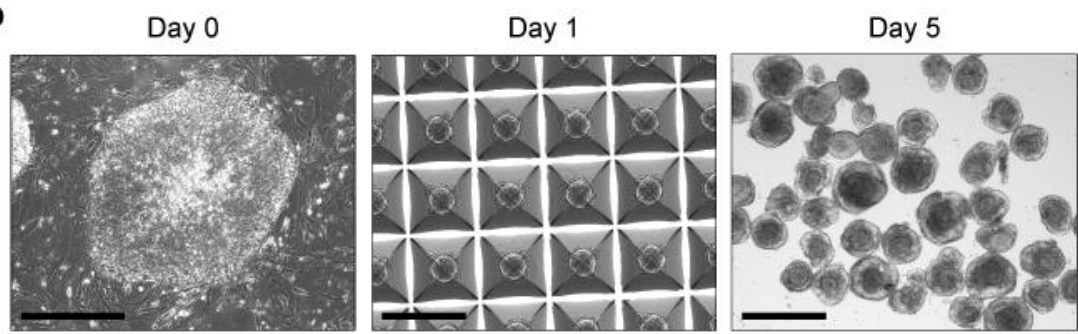

C

Phase-contrast

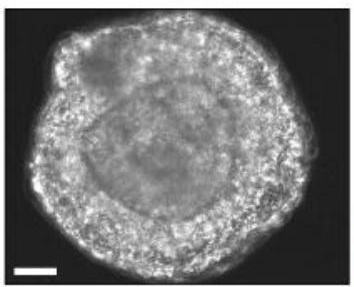

SOX17 OCT4 DAPI

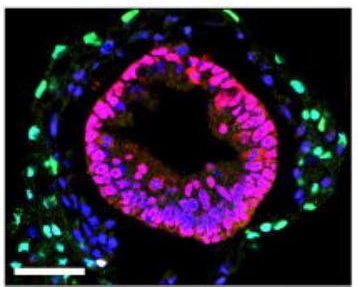

Laminin Glyco-aDG DAPI

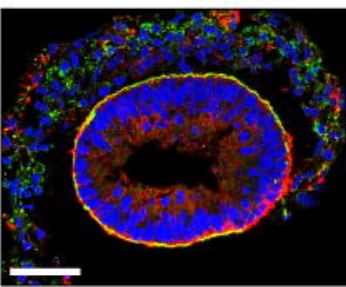


a Serum-replacement medium
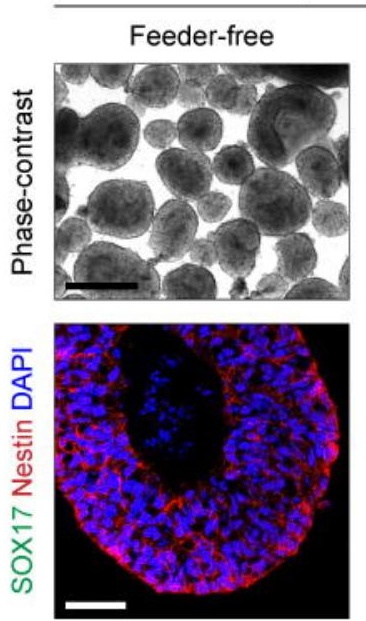

b

\section{Feeder-dependent}
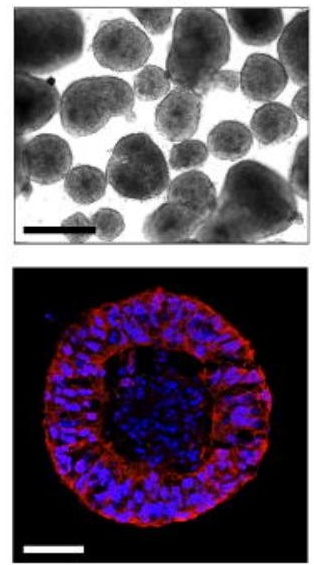

Serum-containing medium

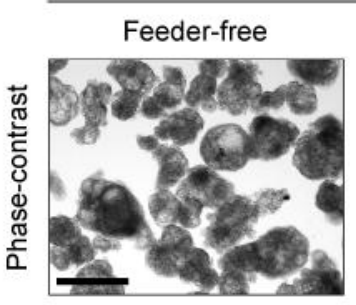

Feeder-dependent
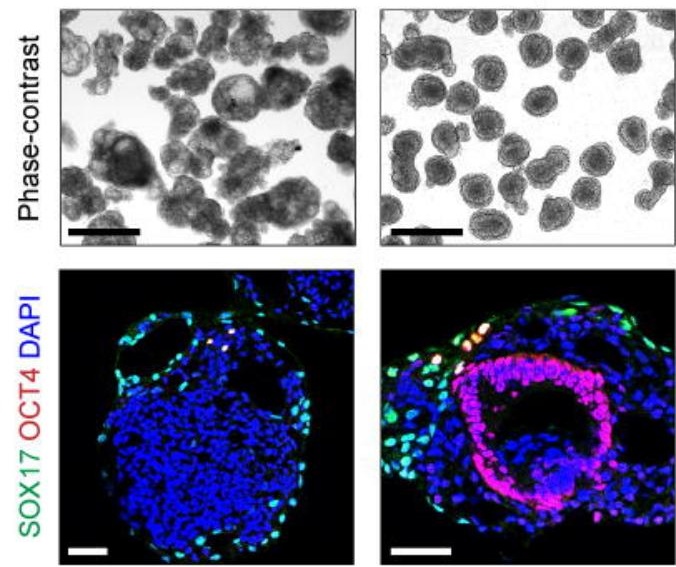
a

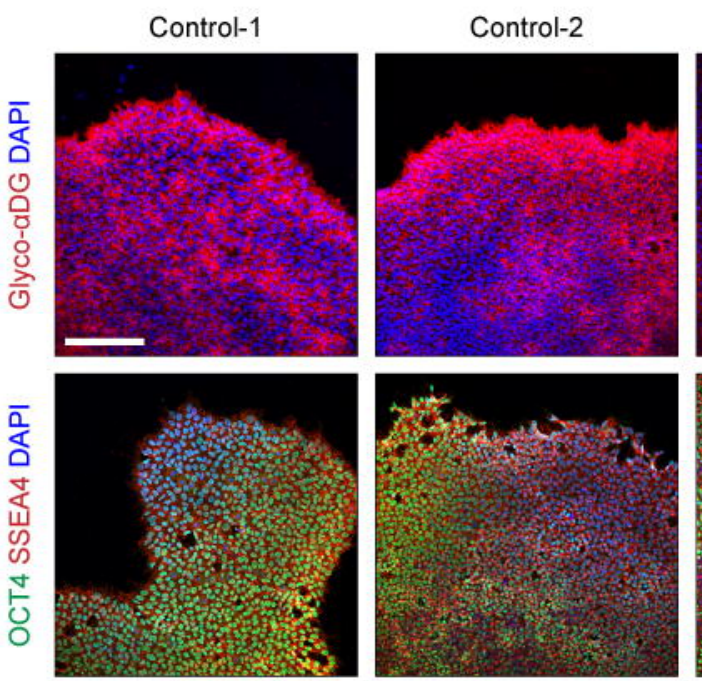

LARGE
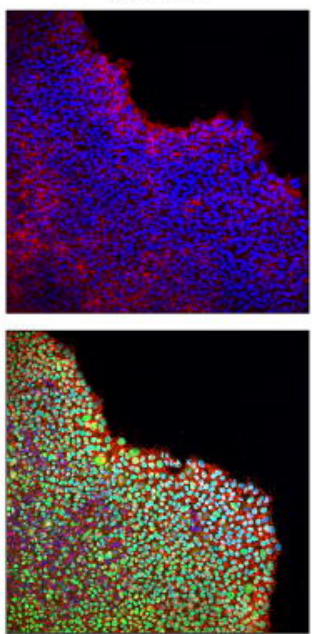

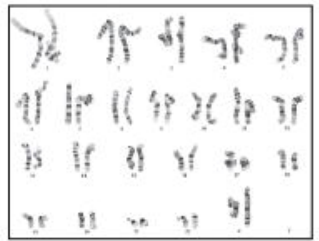

FKRP
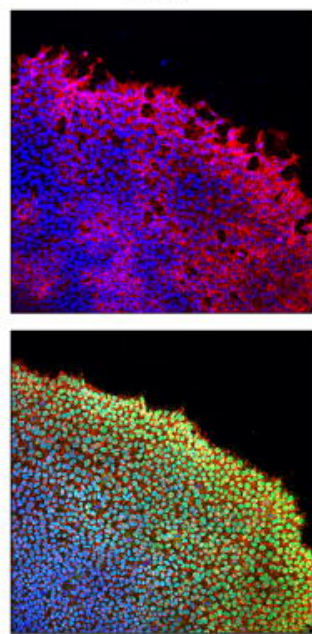

$712 y$ if 10 II g Iit th th is 85 a $0 \mathrm{~g}$.
POMT2

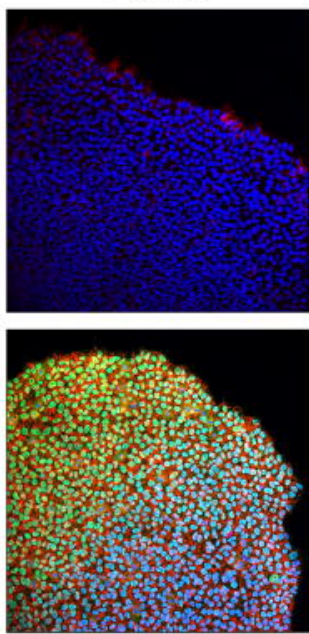

b

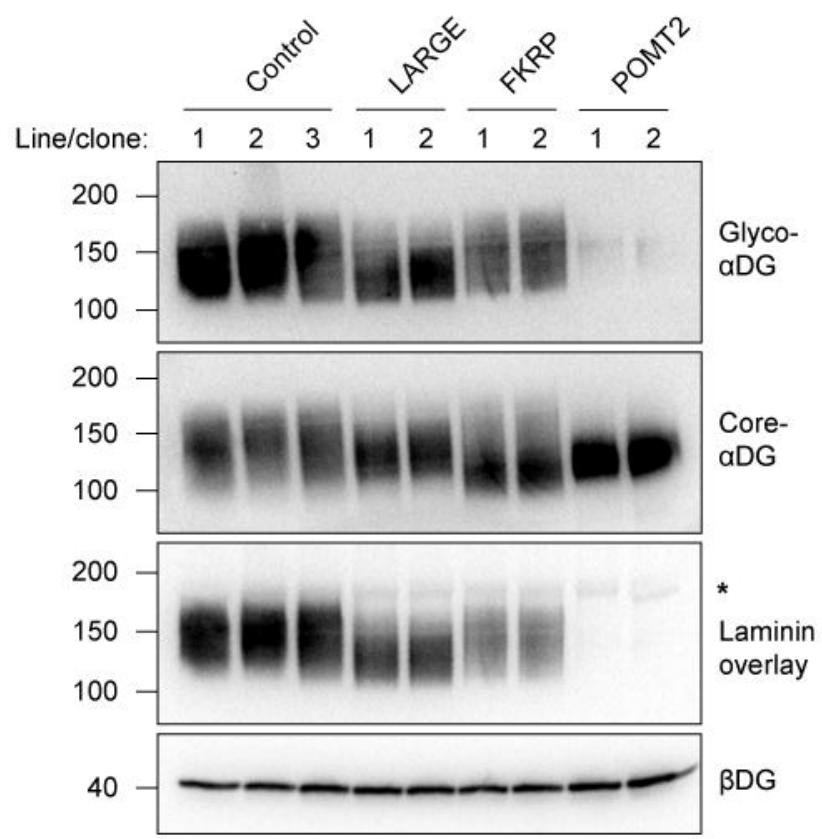

C

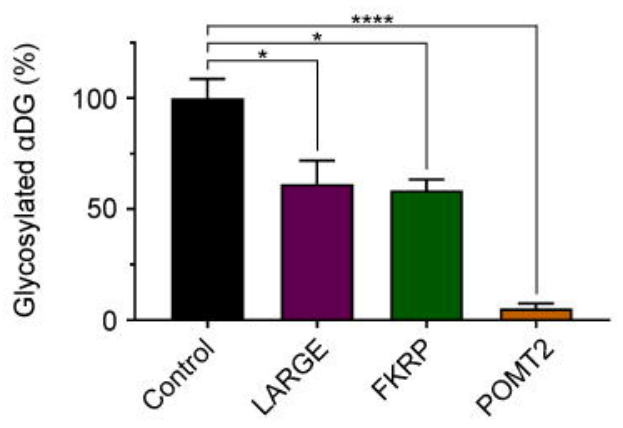

d

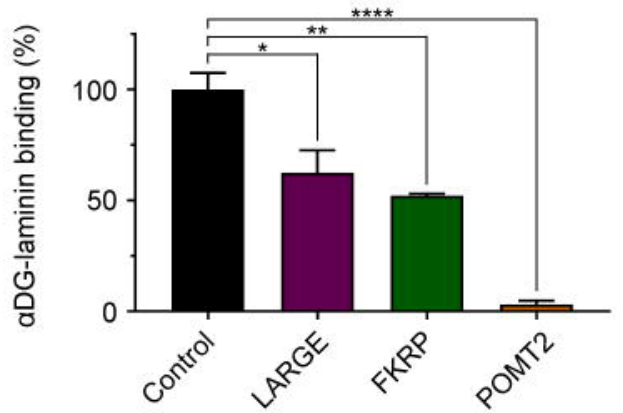


a

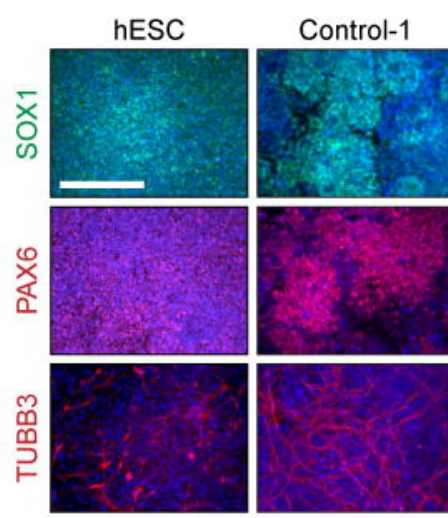

b

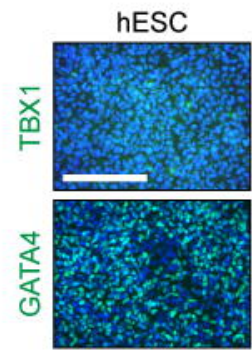

C
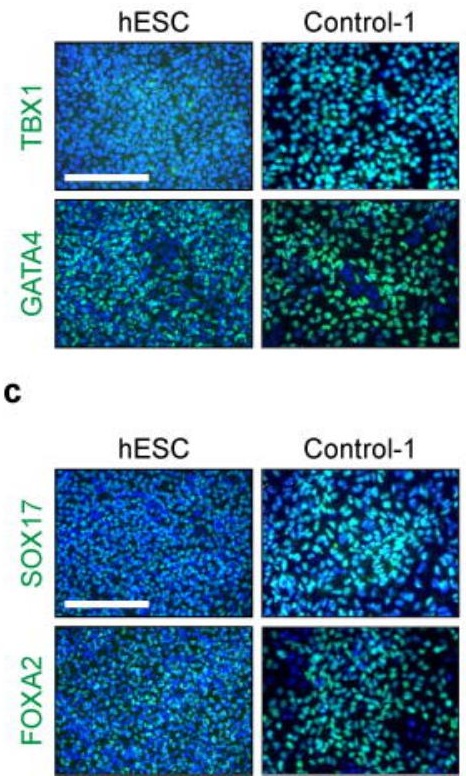

LARGE
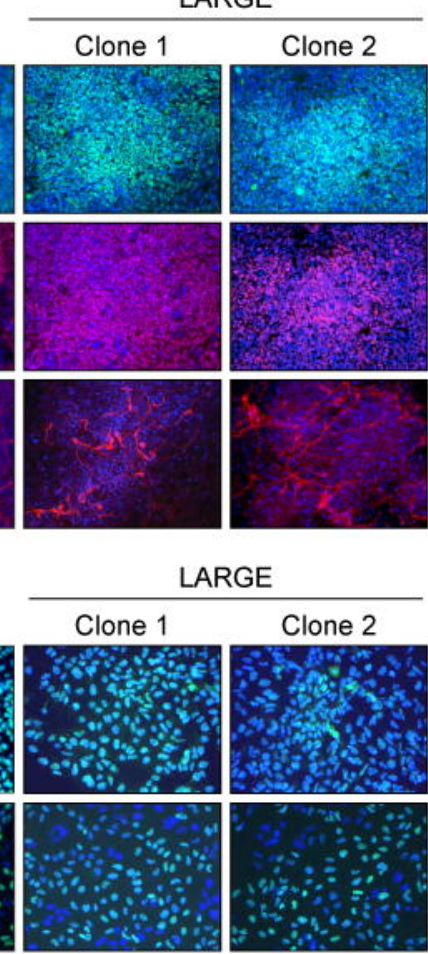

ARGE

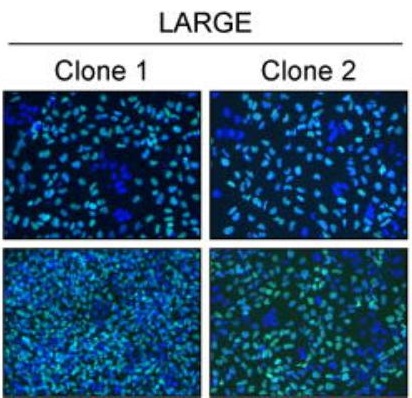

FKRP
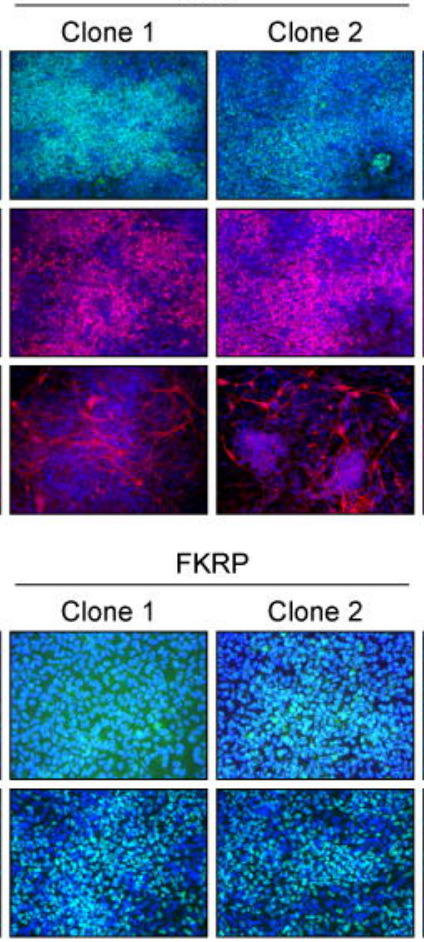

FRP
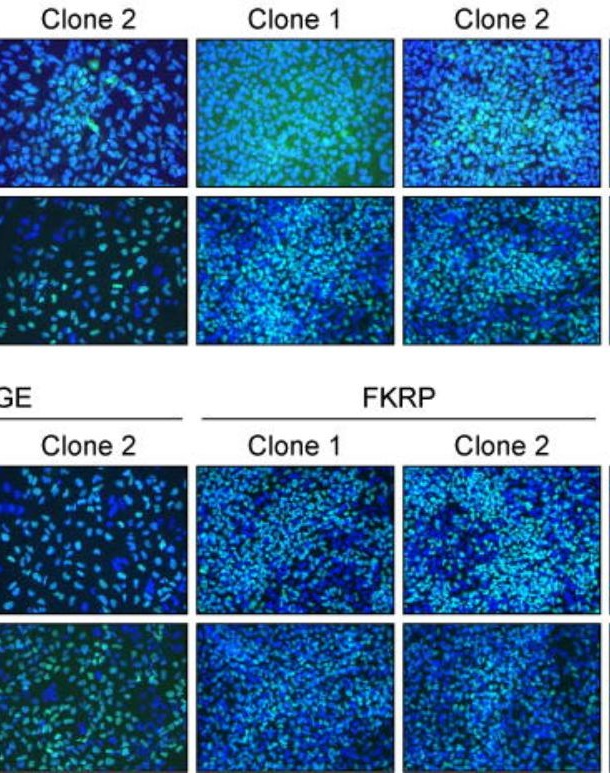

POMT2
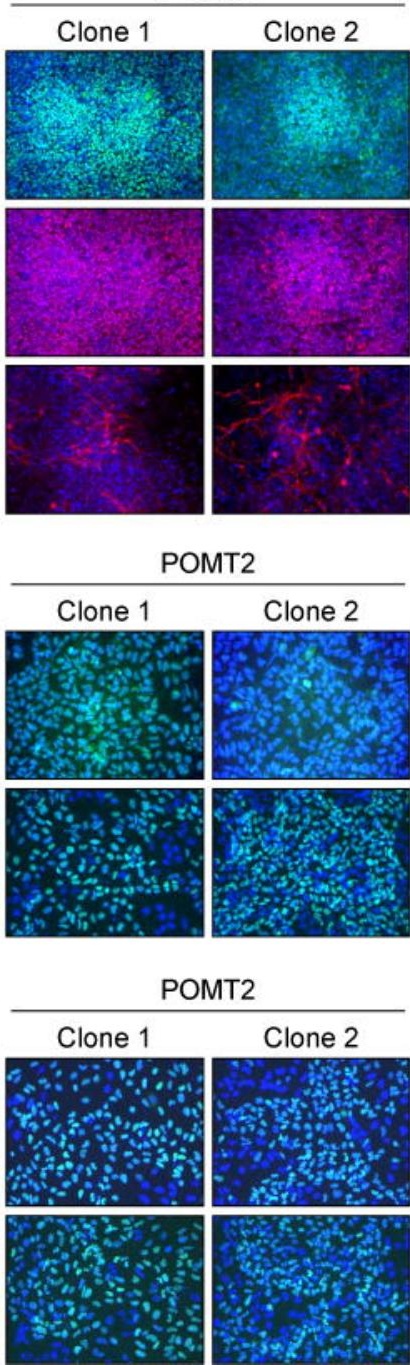
a

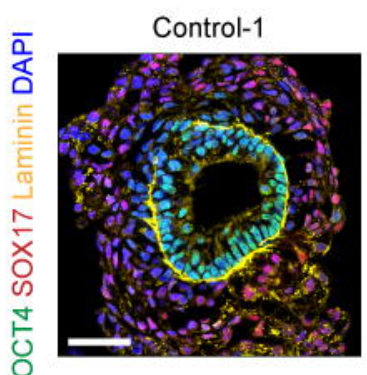

Control-2

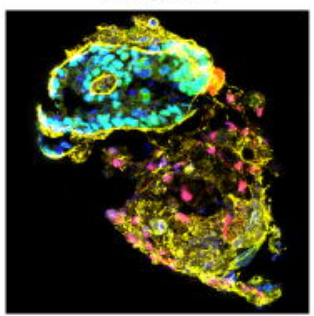

Control-3

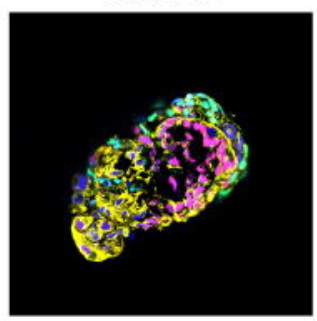

LARGE

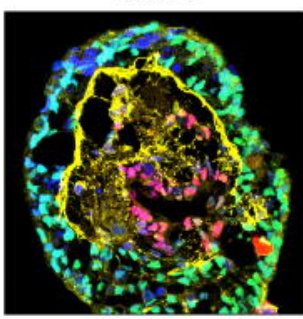

POMT2

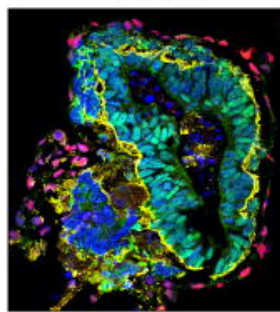

b

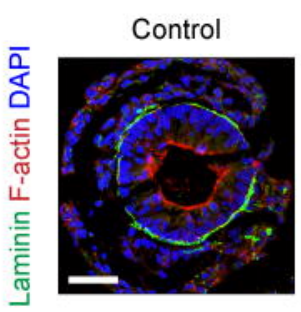

d

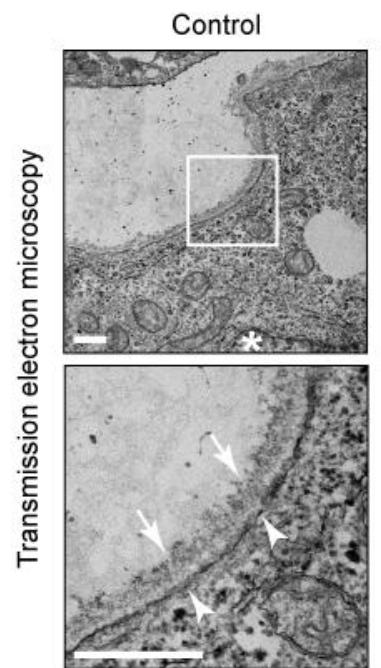

LARGE
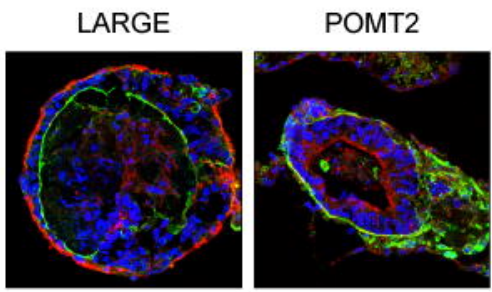

C

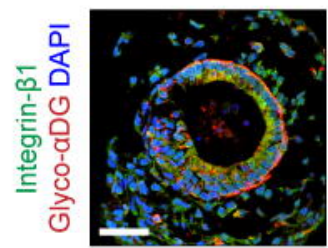

LARGE

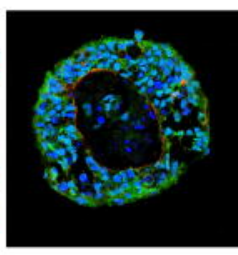

POMT2

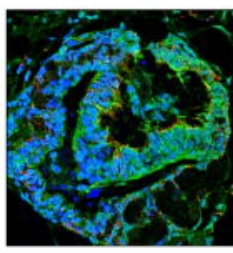

e
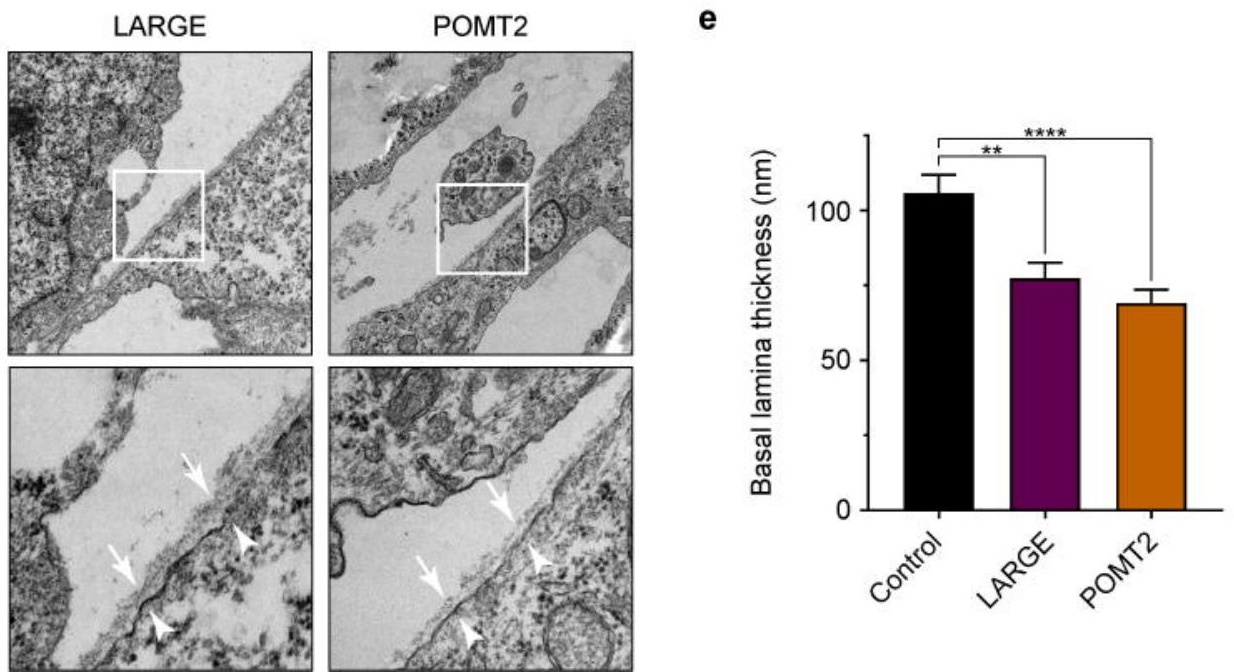
Fig. 6

Control
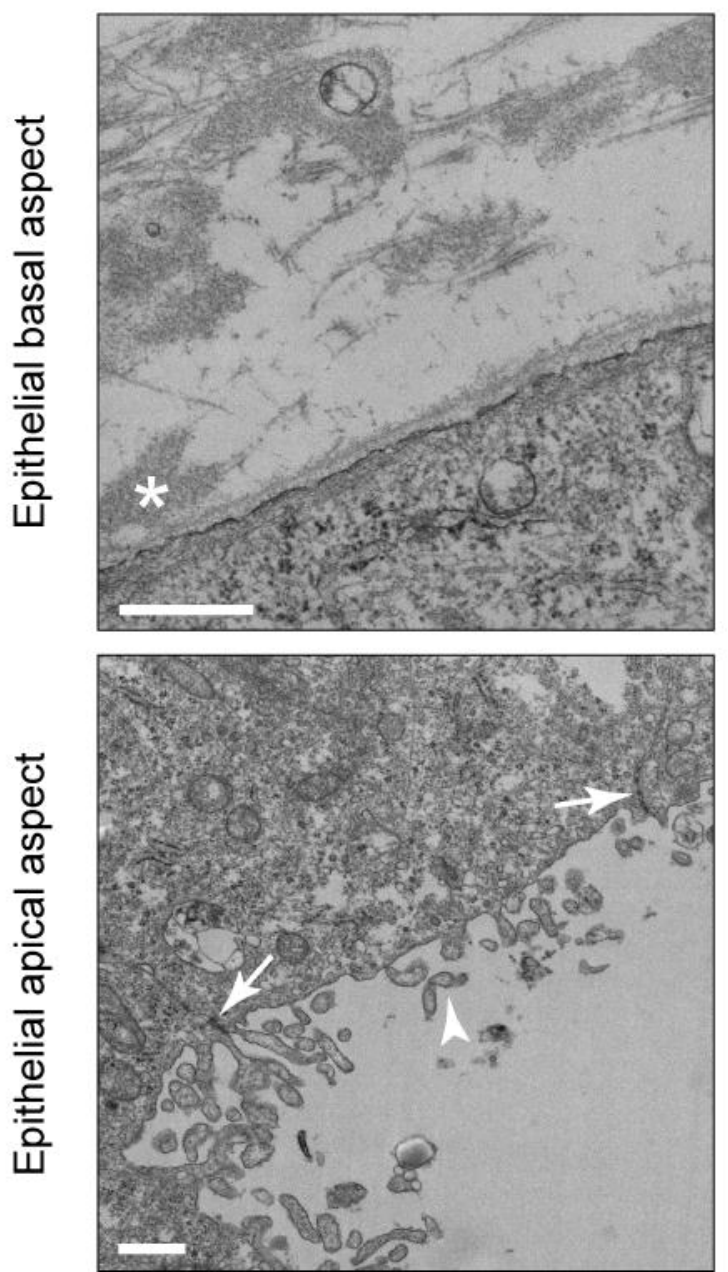

POMT2
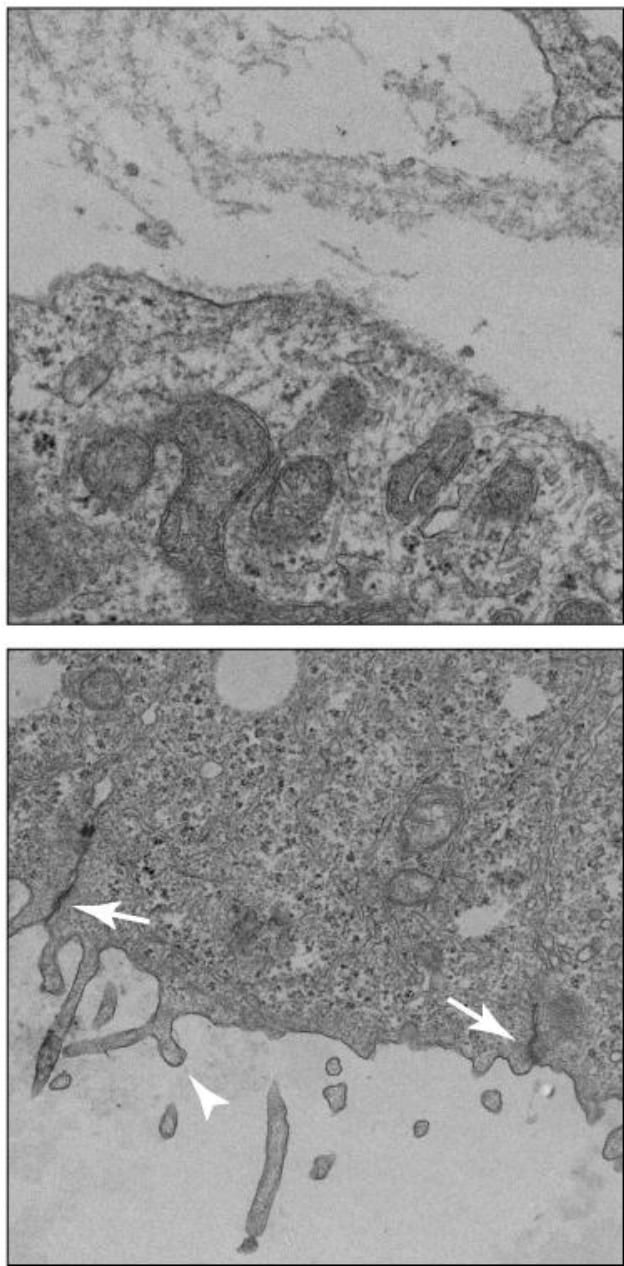
a
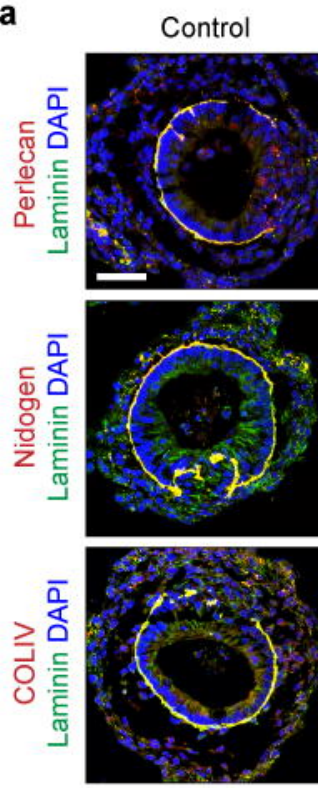

c
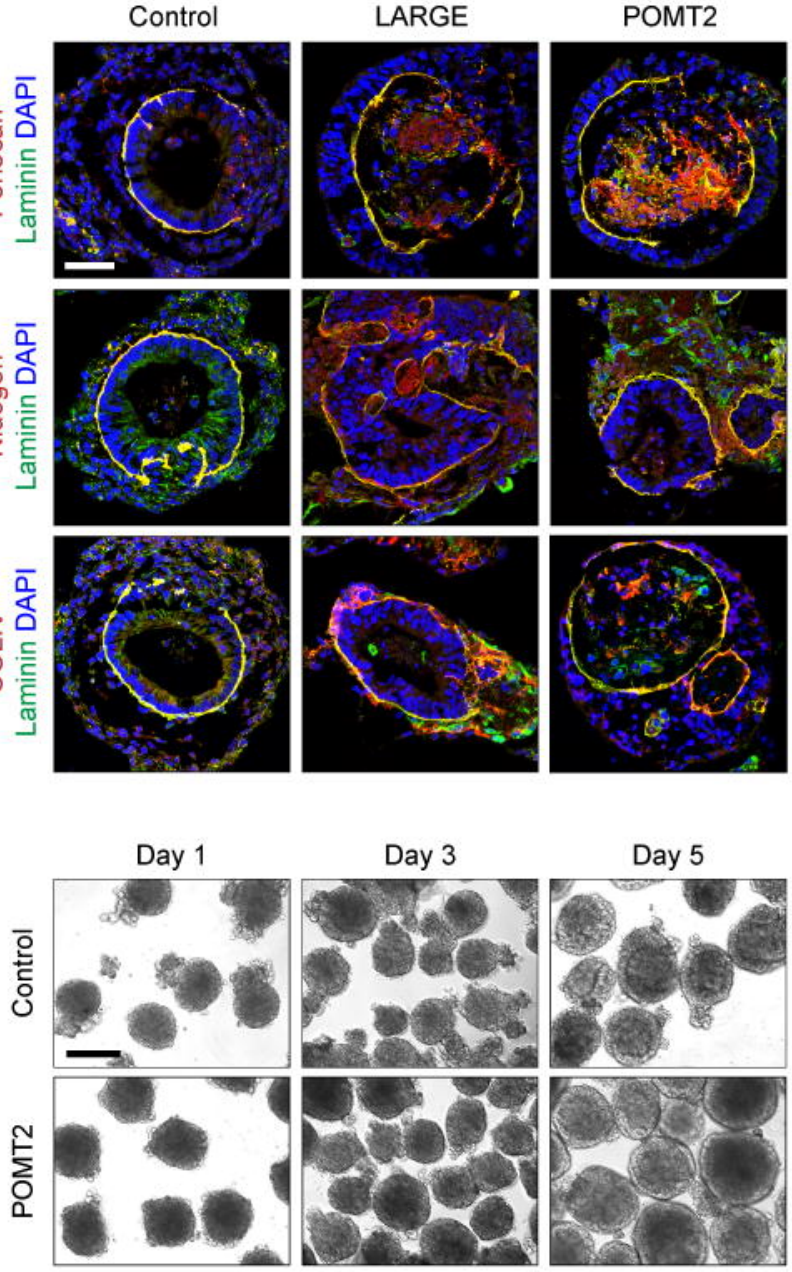

b
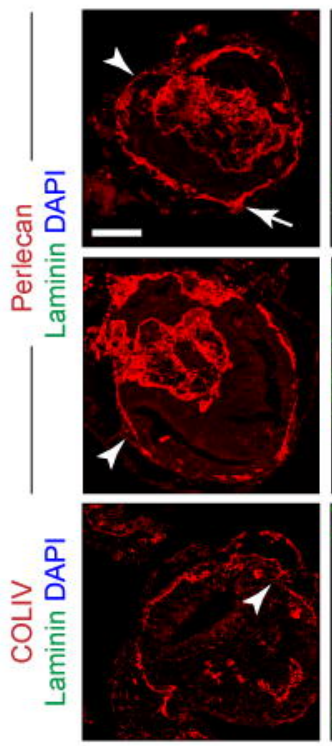

는
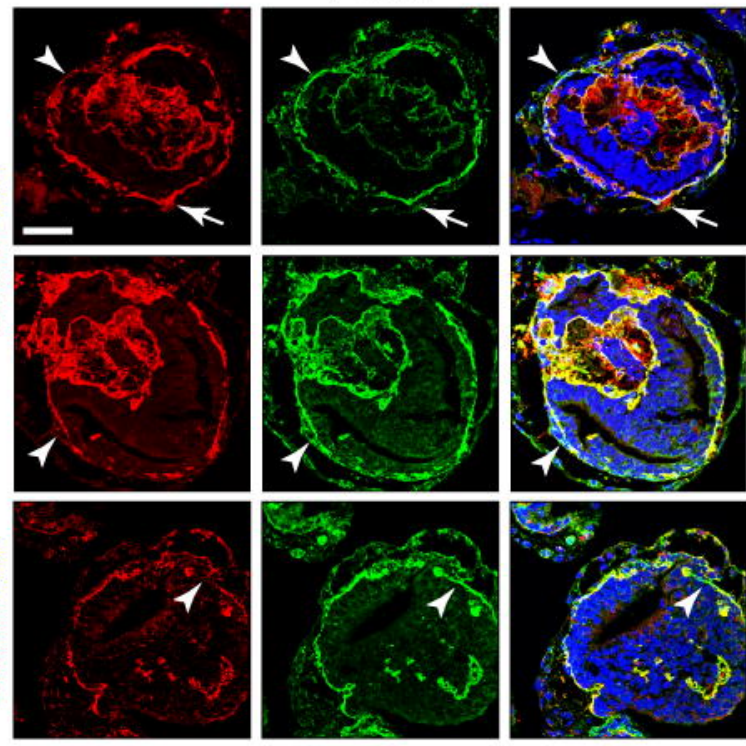

d

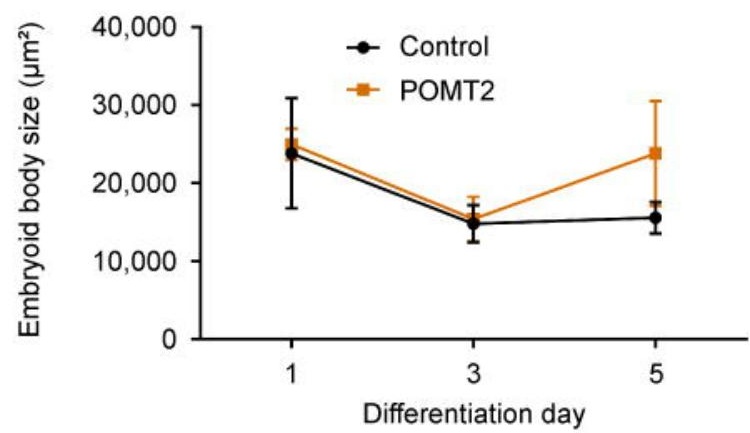


a
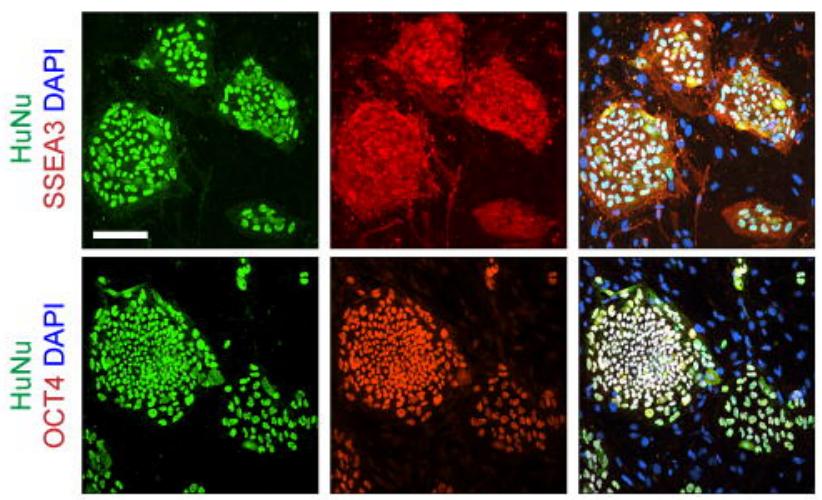

b Control embryoid bodies

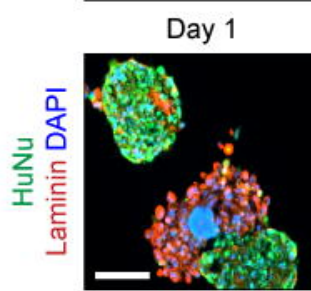

Day 3

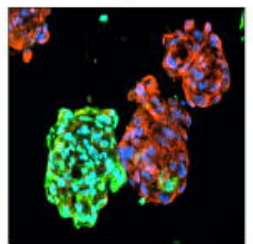

Day 5

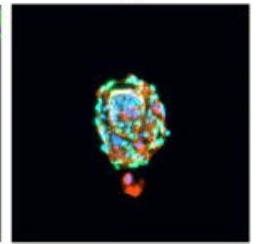

POMT2 iPSCs

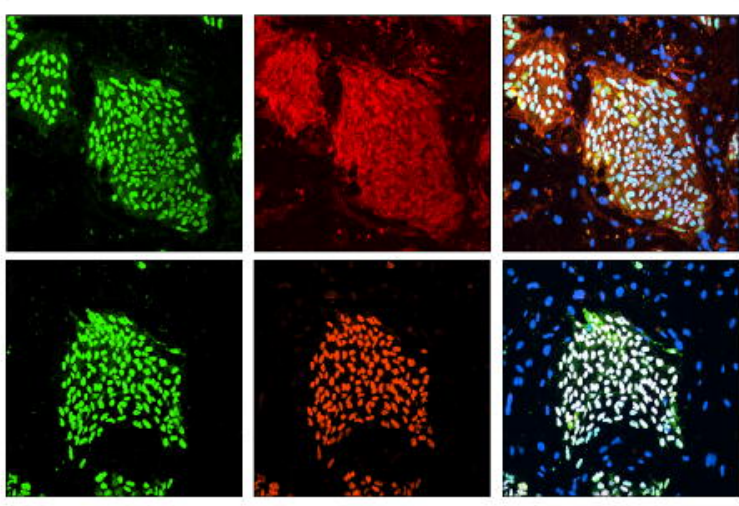

POMT2 embryoid bodies

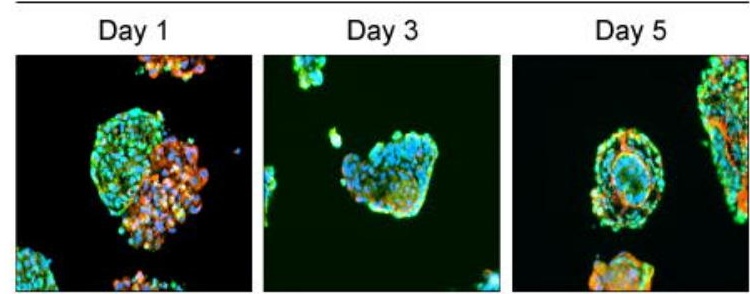


Fig. 9

a

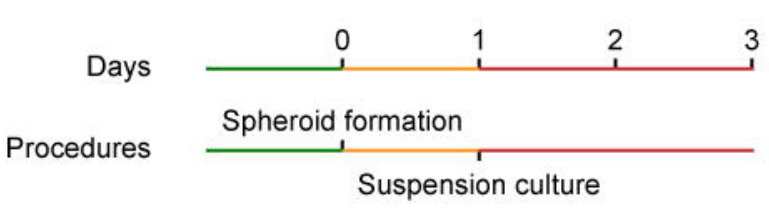

Medium b

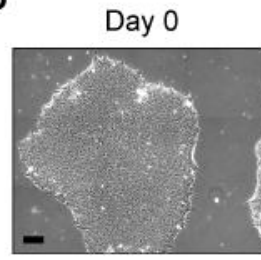

Day 1

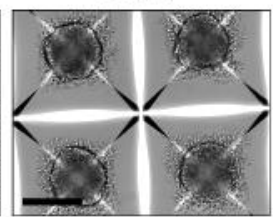

Day 3

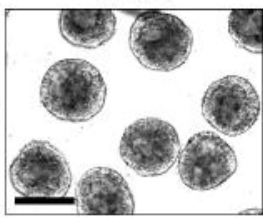

c

Laminin: $\quad 0 \mu \mathrm{g} / \mathrm{mL}$

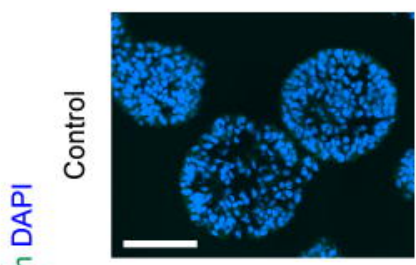

$10 \mu \mathrm{g} / \mathrm{mL}$

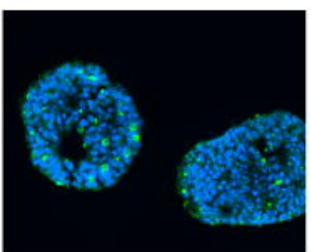

$100 \mu \mathrm{g} / \mathrm{mL}$

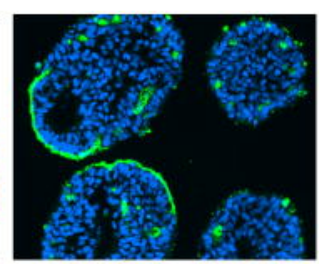

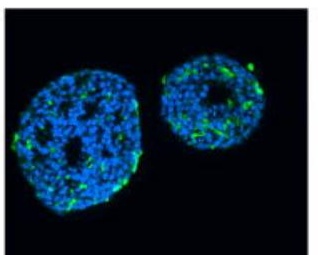

$140 \mu \mathrm{g} / \mathrm{mL}$

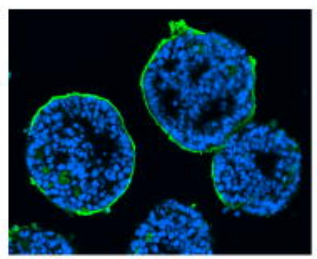

$180 \mu \mathrm{g} / \mathrm{mL}$
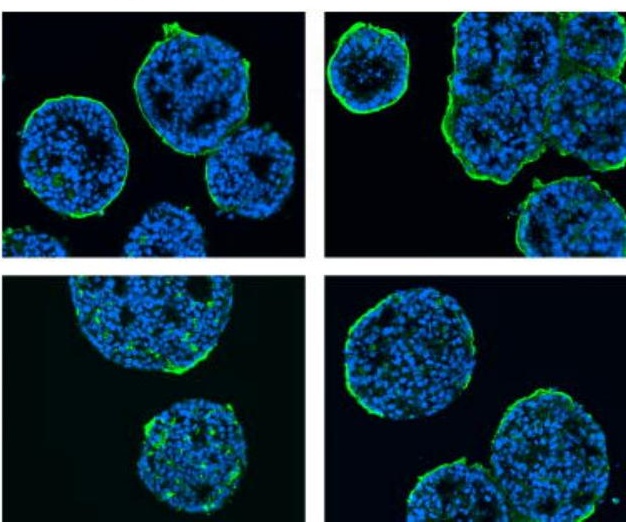
a

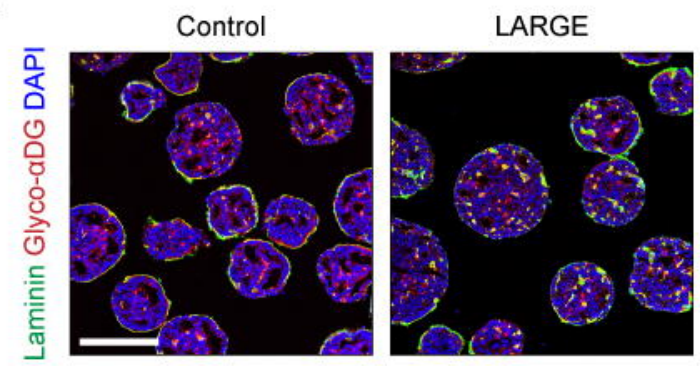

b

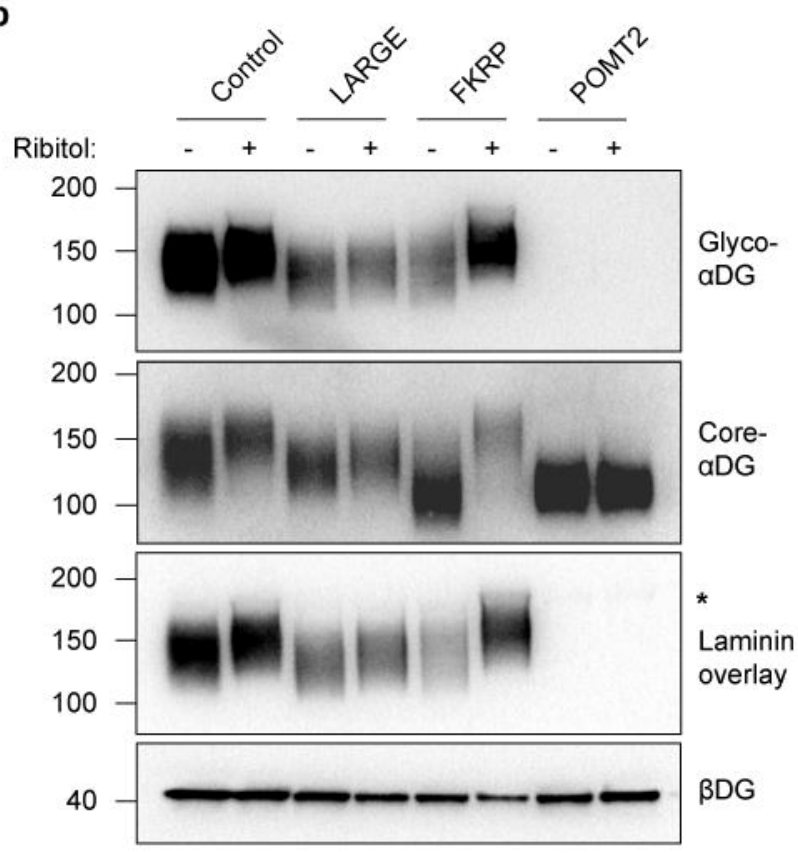

d

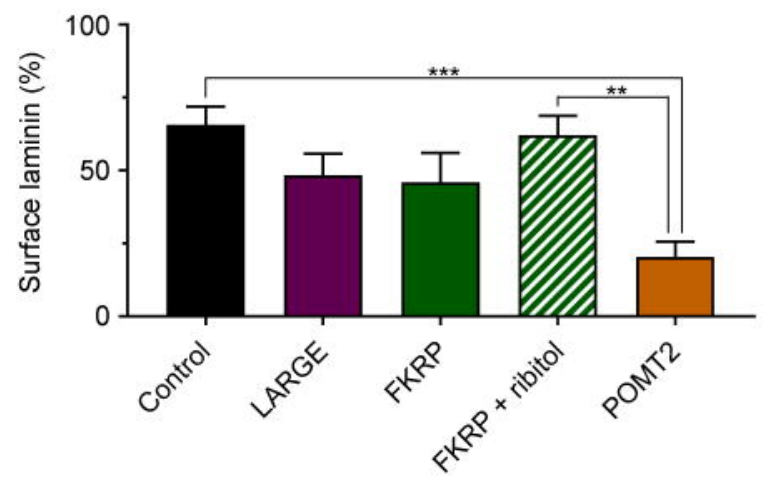

FKRP

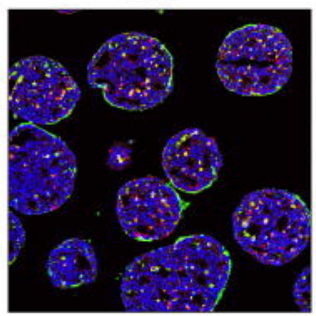

C
FKRP + ribitol

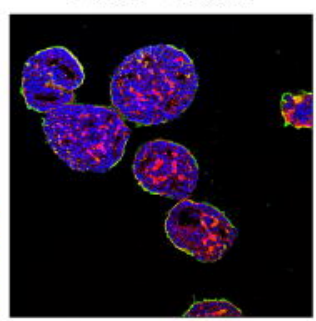

POMT2

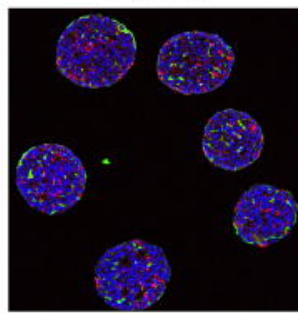

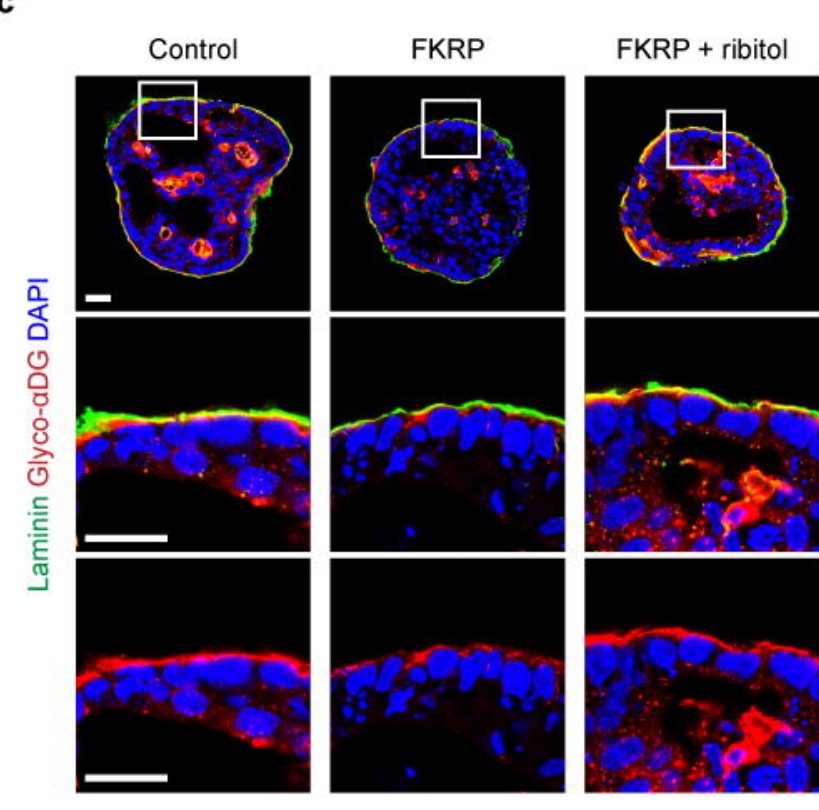

e

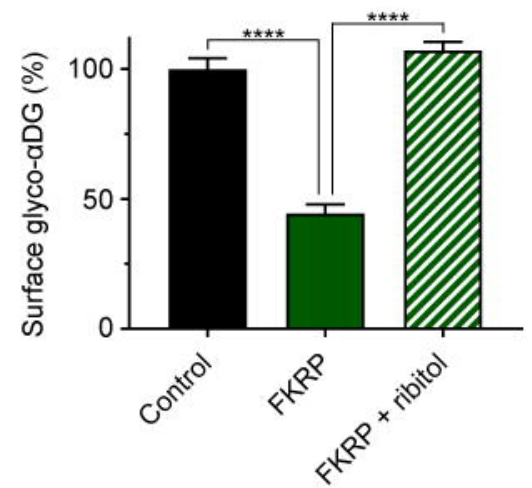


a

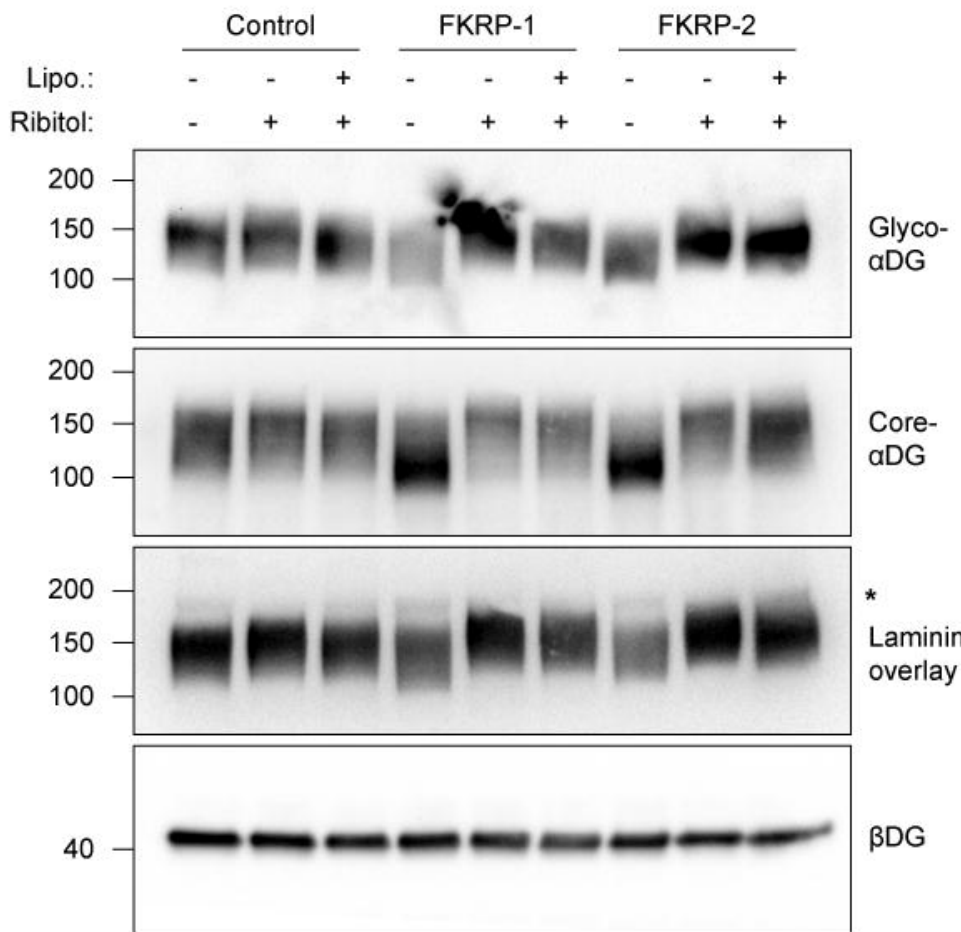

b

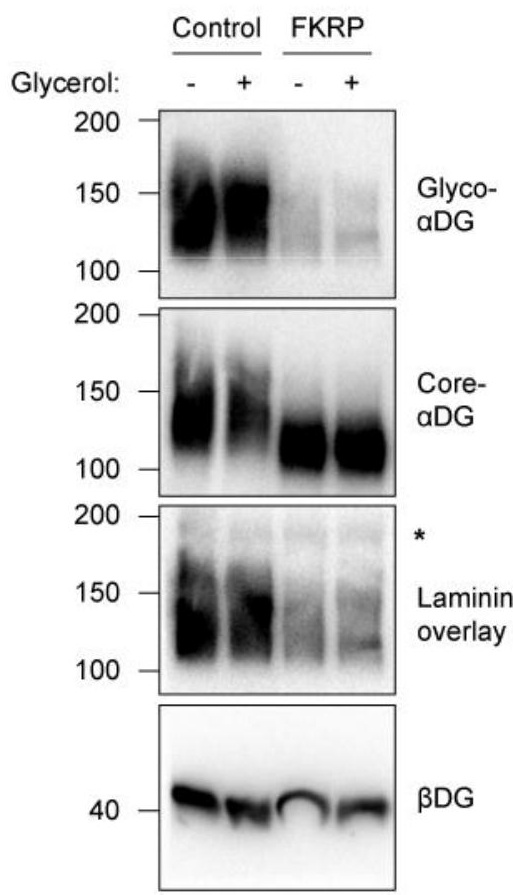

